\title{
Analysis of the kinematic boundaries of the quasielastic neutrino-nucleus cross section in the superscaling model with a relativistic effective mass
}

\author{
I. Ruiz Simo $\odot,{ }^{1, *}$ I. D. Kakorin $\odot,{ }^{2}$ V. A. Naumov $\odot,{ }^{2}$ K. S. Kuzmin $\odot,{ }^{2,3}$ and J. E. Amaro ${ }^{1}$ \\ ${ }^{1}$ Universidad de Granada and Instituto Interuniversitario Carlos I de Física Teórica y Computacional, \\ E-18071 Granada, Spain \\ ${ }^{2}$ Bogoliubov Laboratory of Theoretical Physics, Joint Institute for Nuclear Research, \\ RU-141980 Dubna, Russia \\ ${ }^{3}$ Institute for Theoretical and Experimental Physics, RU-117259 Moscow, Russia
}

(Received 11 February 2021; accepted 10 December 2021; published 4 January 2022)

\begin{abstract}
In this work we obtain the analytical expressions for the boundaries of the charged current quasielastic (CCQE) double differential cross section in terms of dimensionless energy and momentum transfers, for the Relativistic Fermi Gas (RFG) and the Superscaling approach with relativistic effective mass (SuSAM*) models, within the scaling formalism. In addition, we show that this double differential cross section in the scaling formalism has very good properties to be implemented in the Monte Carlo (MC) neutrino event generators, particularly because its peak is almost flat with the (anti)neutrino energy. This makes it especially well suited for the event generation by the acceptance-rejection method usually used in the neutrino generators. Finally, we analyze the total CCQE cross section $\sigma\left(E_{\nu}\right)$ for both models and attribute the enhancement observed in the SuSAM* total cross section to the high-momentum components which are present, in a phenomenological way, in its scaling function, while these are absent in the RFG model.
\end{abstract}

DOI: $10.1103 /$ PhysRevD.105.013001

\section{INTRODUCTION}

The measurement of neutrino/antineutrino-nucleus cross sections is a fundamental topic of research, not only in itself because it can provide knowledge on the fundamental interaction and on the nuclear properties and modeling, but also for its importance in other special fields in particle physics, such as the mixing of neutrino flavors, the extraction of the $C P$-violating phase in the lepton sector and the origin of the asymmetry between matter and antimatter in the Universe. In particular, in the last years many reviews and works have been dedicated to these topics [1-10].

The total integrated Charged Current Quasielastic (CCQE) neutrino/antineutrino cross section $\sigma_{\mathrm{CCQE}}\left(E_{\nu}\right)$ is an important quantity to be known for the neutrino scattering and oscillation experiments [11-35]. In particular, the knowledge of this observable is crucial for choosing of CCQE channel among others to generate appropriate final lepton event kinematics in neutrino event generators,

*uizsig@ugr.es

Published by the American Physical Society under the terms of the Creative Commons Attribution 4.0 International license. Further distribution of this work must maintain attribution to the author(s) and the published article's title, journal citation, and DOI. Funded by SCOAP ${ }^{3}$. that usually use the acceptance-rejection method to generate the events with a probability distribution given by differential cross section.

In addition, the importance of a precise knowledge of the total CCQE cross section and particularly its ratio between the electron and muon neutrinos species is of great importance in order to reduce the systematic uncertainties for the determination of the $C P$-violating phase in the lepton sector, as it has been shown in Refs. [36-42].

Our aim in this work is to perform a thorough study of the analytical boundaries of the phase space of the CCQE double differential cross section $\frac{d^{2} \sigma}{d T_{\mu} d \cos \theta_{\mu}}$ for the relativistic Fermi gas (RFG) [43-47] and superscaling with relativistic effective mass (SuSAM*) models [48-52] within the scaling formalism [53-57], where the boundaries are easier to obtain. To this end, we will study the double differential $\frac{d^{2} \sigma}{d \kappa d \lambda} \mathrm{CCQE}$ cross section, where $\kappa$ and $\lambda$ are the dimensionless momentum and energy transfer variables in the scaling formalism. This new double differential cross section has also the very good property, for the generation of the final charged lepton kinematics in the MC event generators, of an almost flat peak, i.e., very weak dependent on the neutrino/antineutrino energy. This important feature makes it specially well suited for the generation of these events by the acceptance-rejection method. It seems that this fact was already known by some scientists working in the implementation of theoretical models in some MC event 
generators [58], but it was not familiar to us until this article was completed.

The paper is organized as follows: In Sec. II we review in brief the general formalism for the description of the CCQE double differential cross section; in Sec. III we perform a thorough discussion about the analytical boundaries of the phase space in the RFG model, later extended to the SuSAM* model in Sec. IV. In Sec. V we show our main results for the double differential cross section and the integrated total one, and, finally, in Sec. VI we draw our conclusions and outline our future plans or prospects related to the conclusions of the present work.

\section{GENERAL FORMALISM}

In this section, we are going to discuss in brief the elementary ingredients to calculate the double differential CCQE $\frac{d^{2} \sigma}{d T_{\mu} d \cos \theta_{\mu}}$ cross section and its transformation into the easier to work, for our purposes within the scaling formalism, $\frac{d^{2} \sigma}{d \kappa d \lambda}$ cross section. The expression for the first double differential cross section is given by [51,59-61]:

$$
\begin{aligned}
\frac{d^{2} \sigma}{d T_{\mu} d \cos \theta_{\mu}}= & \frac{G_{F}^{2} \cos ^{2} \theta_{c}}{4 \pi} \frac{k^{\prime}}{E_{\nu}} v_{0}\left(V_{\mathrm{CC}} R_{\mathrm{CC}}+2 V_{\mathrm{CL}} R_{\mathrm{CL}}\right. \\
& \left.+V_{\mathrm{LL}} R_{\mathrm{LL}}+V_{T} R_{T} \pm 2 V_{T^{\prime}} R_{T^{\prime}}\right),
\end{aligned}
$$

where $G_{F}=1.116 \times 10^{-11} \mathrm{MeV}^{-2}$ is the Fermi coupling constant, $\theta_{c}$ is the Cabibbo angle $\left(\cos \theta_{c}=0.975\right), k^{\prime}$ is the value of the final charged lepton momentum, $\vec{k}^{\prime}, E_{\nu}$ is the neutrino/antineutrino energy in the lab frame, and $v_{0}=\left(E_{\nu}+T_{\mu}+m_{\mu}\right)^{2}-q^{2}$, with $q^{2}$ being the squared three-momentum transfer, $\vec{q}$, to the nucleus [62]. Finally, it is worth noting that the \pm sign in the $T^{\prime}$ contribution of Eq. (1) applies for neutrino and antineutrino CCQE scattering, respectively.

The other ingredients appearing in Eq. (1) are the lepton kinematic factors $V_{K}$ and the nuclear response functions $R_{K}$, the last ones depending only on the energy and momentum transfer from the leptons to the nucleus, $\omega$ and $q$, respectively. These factors come mainly from the contraction of the lepton tensor with the hadron one, and each of them are suitable combinations of the tensors in a frame where the $Z$-axis is defined by the direction of the three-momentum transfer, $\vec{q}=\vec{k}-\vec{k}^{\prime}$. Their explicit expressions can be found, for instance, in Refs. [51,59-61], and particularly in Sec. IIIA and Appendices B and C of the recent review [8], where an exhaustive discussion and derivation of the response functions and scaling in the RFG model are given.

It is quite general that the nuclear response functions $R_{K}$ can be written in factorized form as a product of an integrated single-nucleon response $\left(U_{K}\right.$ or $G_{K}$ in the nomenclature of Ref. [8]) times a scaling function which depends on the nuclear model. Nonetheless, in other nuclear models different from those discussed in this work, several different scaling functions can appear for the different nuclear response functions. Examples of these are the models for the description of the $\mathrm{QE}$ response in the inclusive $\left(e, e^{\prime}\right)$ or $(\nu, \mu)$ scattering, where different scaling functions appear for each one of the nuclear responses (see Refs. [55,63,64], just to cite a few of them).

However, in the two models discussed in this work, a single scaling function appears as a common factor in all the nuclear response functions $R_{K}$ and factorizes in the cross section given in Eq. (1):

$$
\begin{aligned}
\frac{d^{2} \sigma}{d T_{\mu} d \cos \theta_{\mu}}= & \frac{G_{F}^{2} \cos ^{2} \theta_{c}}{4 \pi} \frac{k^{\prime}}{E_{\nu}} v_{0}\left(V_{\mathrm{CC}} U_{\mathrm{CC}}+2 V_{\mathrm{CL}} U_{\mathrm{CL}}\right. \\
& \left.+V_{\mathrm{LL}} U_{\mathrm{LL}}+V_{T} U_{T} \pm 2 V_{T^{\prime}} U_{T^{\prime}}\right) f_{\mathrm{scal}}(\psi),
\end{aligned}
$$

where $\psi$ is the scaling variable and it is, in general, a function of $\omega$ and $q$. For instance, in the particular case of the RFG model, its expression is given by

$$
f_{\mathrm{RFG}}(\psi)=\frac{3}{4}\left(1-\psi^{2}\right) \theta\left(1-\psi^{2}\right),
$$

where $\theta(x)$ is the step function, while in the SuSAM* model its expression is discussed in Sec. IV.

However, the differential cross section of Eq. (1) is given with respect to the final lepton kinematic variables, its kinetic energy $T_{\mu}$ and the cosine of its scattering angle with respect to the incident neutrino direction, $\theta_{\mu}$. In the scaling formalism it is not very difficult to find the relevant boundaries where the differential cross section of Eq. (1) is different from zero (as it will be shown in Secs. III and IV, and Appendices A and B), but using the relevant scaling variables, namely, the dimensionless energy and momentum transfers, $\lambda=\omega /\left(2 m_{N}\right)$ and $\kappa=q /\left(2 m_{N}\right)$, with $m_{N}$ the nucleon mass.

Therefore, in order to inspect the behavior of the double differential cross section along its phase space and efficiently integrate it to obtain the total CCQE cross section, it is better to work with the $\frac{d^{2} \sigma}{d \kappa d \lambda}$ cross section, which can be obtained from that in Eq. (1) using the Jacobian transformation from $\left(T_{\mu}, \cos \theta_{\mu}\right)$ variables to $(\kappa, \lambda)$ ones:

$$
\begin{aligned}
\frac{d^{2} \sigma}{d \kappa d \lambda} & =\left|\frac{\partial\left(T_{\mu}, \cos \theta_{\mu}\right)}{\partial(\kappa, \lambda)}\right| \frac{d^{2} \sigma}{d T_{\mu} d \cos \theta_{\mu}} \\
& =\frac{4 m_{N}^{2} q}{E_{\nu} k^{\prime}} \frac{d^{2} \sigma}{d T_{\mu} d \cos \theta_{\mu}},
\end{aligned}
$$

where the Jacobian has been calculated knowing the relationships between both sets of independent variables, 


$$
\begin{aligned}
& \lambda=\frac{E_{\nu}-T_{\mu}-m_{\mu}}{2 m_{N}} \\
& \kappa=\frac{\sqrt{E_{\nu}^{2}+P_{\mu}^{2}-2 E_{\nu} P_{\mu} \cos \theta_{\mu}}}{2 m_{N}},
\end{aligned}
$$

with $E_{\mu}=T_{\mu}+m_{\mu}$ and $P_{\mu} \equiv k^{\prime}=\sqrt{E_{\mu}^{2}-m_{\mu}^{2}}$.

\section{RFG MODEL CASE}

\section{A. Analytical boundaries due to the scaling model}

With the definitions of the dimensionless variables in the scaling formalism, where the electroweak probe transfers energy $\omega$ and momentum $q$ to the nucleus:

$$
\begin{gathered}
\lambda=\frac{\omega}{2 m_{N}}, \quad \kappa=\frac{q}{2 m_{N}} \\
\tau=\kappa^{2}-\lambda^{2}=\frac{Q^{2}}{4 m_{N}^{2}} \geq 0 \\
\eta_{F}=\frac{k_{F}}{m_{N}}, \quad \epsilon_{F}=\sqrt{1+\eta_{F}^{2}} \geq 1 \\
\psi=\sqrt{\frac{\epsilon_{0}-1}{\epsilon_{F}-1}} \operatorname{sign}(\lambda-\tau),
\end{gathered}
$$

where $\epsilon_{0}$ is defined as

$$
\epsilon_{0}=\max \left(\kappa \sqrt{1+\frac{1}{\tau}}-\lambda, \quad \epsilon_{F}-2 \lambda\right),
$$

and $k_{F}$ is the Fermi momentum of the nucleus. The definition of $\epsilon_{0}$ given in Eq. (7) represents the minimum energy of the initial nucleon, in units of the nucleon mass $m_{N}$, that can contribute to a quasielastic (QE) scattering event for given energy and momentum transfers $(\lambda, \kappa)$ (cf. Eq. (C11) of Ref. [8]).

In the RFG model, see Eq. (3), the scaling variable $\psi$ is restricted to lie between -1 and +1 to get a nonvanishing contribution to the cross section. The scaling variable is zero when $\epsilon_{0}=1$, i.e., when $\lambda=\tau$ (see Appendix A) in the non-Pauli blocking (NPB) region. This condition is equivalent to

$$
\tau=\lambda \Leftrightarrow \kappa^{2}=\lambda^{2}+\lambda \Leftrightarrow \kappa=\sqrt{\lambda(\lambda+1)},
$$

and hence Eq. (8) corresponds to where the scaling variable is always zero, and where the QE peak appears. For this reason, we call this curve in the $(\lambda, \kappa)$ plane as $\kappa_{\mathrm{QE}}(\lambda)=\sqrt{\lambda(\lambda+1)}$.

The boundaries of the RFG scaling variable $(-1,+1)$ are reached when $\epsilon_{0}=\epsilon_{F}$ as it follows from Eq. (6). Solving the equation $\epsilon_{0}=\kappa \sqrt{1+1 / \tau}-\lambda=\epsilon_{F}$ in the NPB region (corresponding to $\kappa \geq \eta_{F}$ ) we get two different curves in the $(\lambda, \kappa)$ plane. These curves are labeled as $\kappa_{ \pm}^{\mathrm{NPB}}(\lambda)$, and along them the scaling variable is always $\psi=\mp 1$, respectively. For a more detailed derivation the reader is referred to Appendices A 1 and A 2.

The expressions of these two curves are given by

$$
\kappa_{ \pm}^{\mathrm{NPB}}(\lambda)=\frac{1}{2} \sqrt{\left(\epsilon_{F}+2 \lambda\right)^{2}-1} \pm \frac{\eta_{F}}{2}
$$

and they are depicted in Fig. 1.

In Fig. 2 we show the values taken by the scaling variable $\psi(\kappa(\lambda), \lambda)$, as a function of $\lambda$, along different curves in the NPB region. It can be seen that the scaling variable is zero along the curve $\kappa_{\mathrm{QE}}(\lambda)$, i.e., the position of the QE peak. Along the curves $\kappa_{\mp}^{\mathrm{NPB}}(\lambda)$, the scaling variable always takes its limiting values in the RFG model, $\psi= \pm 1$, respectively. These values are shown by the solid and short-dashed lines in Fig. 2, respectively. Any curve lying in between $\kappa_{-}^{\mathrm{NPB}}(\lambda)$ and $\kappa_{\mathrm{QE}}(\lambda)$ in Fig. 1, as the dot-dashed one indicates in Fig. 2, has a positive value for the scaling variable; while those curves lying in between $\kappa_{\mathrm{QE}}(\lambda)$ and $\kappa_{+}^{\mathrm{NPB}}(\lambda)$ always have negative values for the scaling variable, as can be inspected from the dotted line of Fig. 2.

When $\kappa \geq \eta_{F}$ we are in the NPB region and $\epsilon_{0}$ is always equal to the first argument of the maximum function given in Eq. (7). However, when $\kappa<\eta_{F}$, there are some regions in the $(\lambda, \kappa)$-plane where $\epsilon_{0}$ is equal to the second argument of the maximum function of Eq. (7), and we call this region the Pauli blocking (PB) region; while there are other regions where $\epsilon_{0}$ is still equal to the first argument of the maximum function. For a detailed derivation of the boundaries of these regions and other proofs, the reader

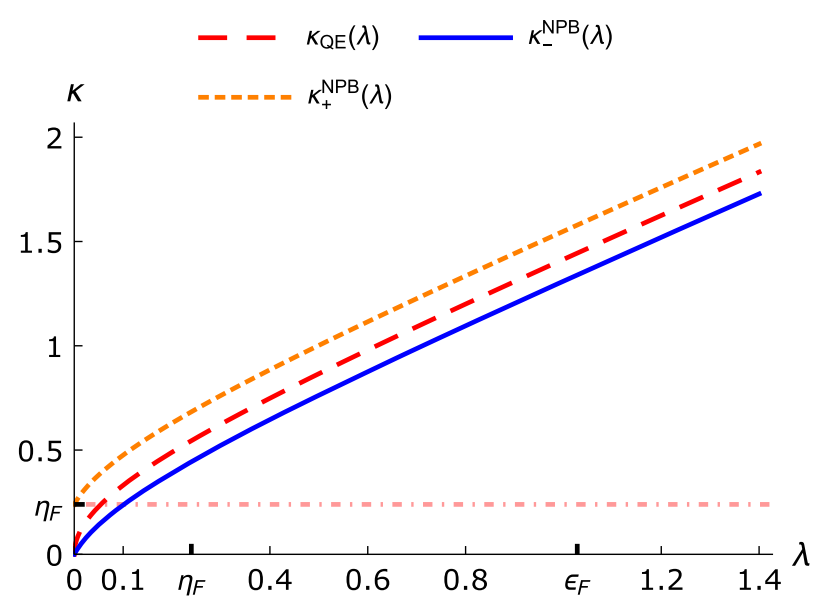

FIG. 1. Plot of the two limiting curves $\kappa_{ \pm}^{\mathrm{NPB}}(\lambda)$ as a function of $\lambda$ in the RFG model in the NPB region, i.e., for $\kappa \geq \eta_{F}$ (notice that for $\kappa<\eta_{F}$ we are entering in the Pauli blocking (PB) region). In this figure, we have taken $\eta_{F}=0.239$. The long-dashed curve corresponds to $\kappa_{\mathrm{QE}}(\lambda)$. 

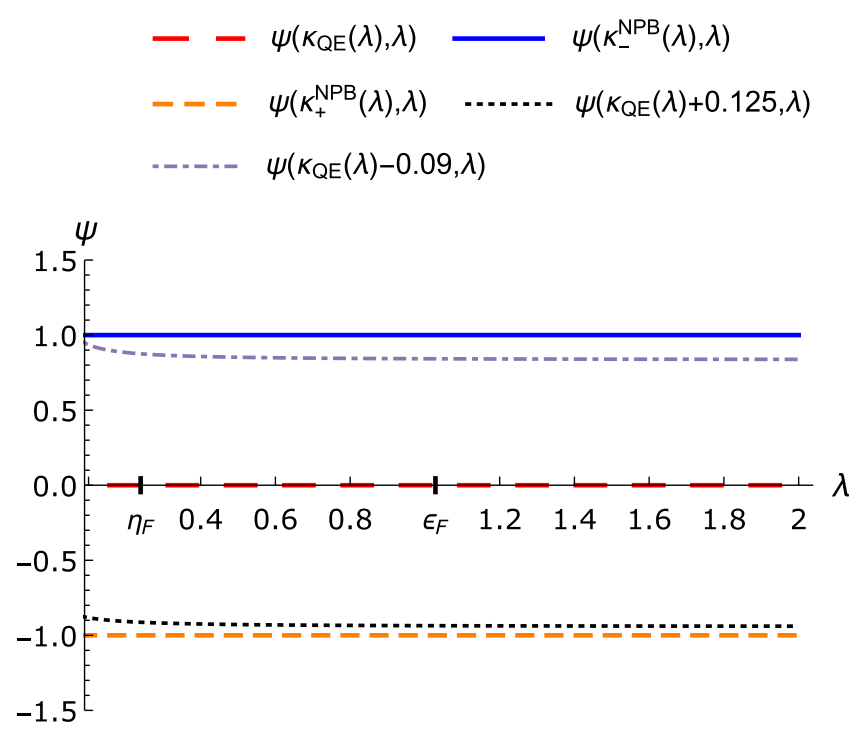

FIG. 2. Plot of the values taken by the scaling variable $\psi$, defined on Eq. (6), as a function of $\lambda$, along different curves in the $(\lambda, \kappa)$-plane. The long-dashed line corresponds to the case $\lambda=\tau$, the solid and short-dashed lines correspond, respectively, to the lower and upper bounds of the RFG, given by $\kappa=\kappa_{\mp}^{\mathrm{NPB}}(\lambda)$ in Fig. 1. Two additional curves are shown for comparison (see main text for discussion).

is referred to Appendix A 3. Here we only provide the final results.

According to the derivation discussed in Appendix A 3, we can conclude that the region where $\mathrm{PB}$ makes $\epsilon_{0}$ to be equal to the second argument of Eq. (7), $\epsilon_{F}-2 \lambda$, corresponds to the region $\kappa_{-}^{\mathrm{PB}}(\lambda) \leq \kappa \leq \kappa_{+}^{\mathrm{PB}}(\lambda)$ in the range where $0 \leq \lambda \leq \lambda_{-}$, with

$$
\kappa_{ \pm}^{\mathrm{PB}}(\lambda)=\sqrt{\frac{\rho \pm \sqrt{\rho^{2}-4\left(\lambda \epsilon_{F}-\lambda^{2}\right)^{2}}}{2}},
$$

where $\rho=2 \lambda^{2}-2 \lambda \epsilon_{F}+\eta_{F}^{2}$ and $\lambda_{-}=\frac{\epsilon_{F}-1}{2}$.

In Fig. 3 we show the different regions filled with colors for $\kappa<\eta_{F}$, where PB effect occurs or not. The shaded regions between $\kappa_{+}^{\mathrm{PB}}(\lambda)$ and $\kappa=\eta_{F}$, and between $\kappa_{-}^{\mathrm{PB}}(\lambda)$ and $\kappa_{-}^{\mathrm{NPB}}(\lambda)$, respectively, correspond to those zones of the allowed phase space of the RFG where there is no PB, i.e., where $\epsilon_{0}=\kappa \sqrt{1+1 / \tau}-\lambda$. On the other hand, the shaded regions between $\kappa_{+}^{\mathrm{PB}}(\lambda)$ and $\kappa_{\mathrm{QE}}(\lambda)$, and between this last curve and the dotted $\kappa_{-}^{\mathrm{PB}}(\lambda)$ one, respectively, correspond to zones where $\epsilon_{0}=\epsilon_{F}-2 \lambda$, i.e., where there is $\mathrm{PB}$. It is worth noting that in this region, delimited by that kind of inverted parabola formed by joining together the dotted and the three-fold dashed curves of Fig. 3, the variable $\epsilon_{0}$ and, consequently, the scaling variable $\psi$ only depend on $\lambda$ and not at all on $\kappa$. The only important issue to select the sign of $\psi$ is whether the points in these regions are above the long-dashed thick line corresponding to the curve

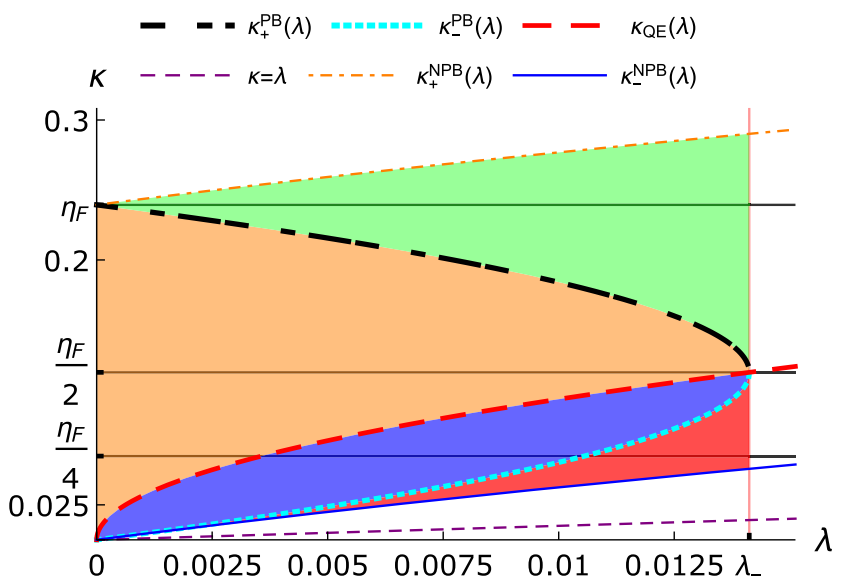

FIG. 3. Plot of the $(\lambda, \kappa)$-plane in the PB region, i.e., for $0 \leq$ $\lambda \leq \lambda_{-}$and $0<\kappa<\eta_{F}$. The three-fold dashed thick curve corresponds to the $\kappa_{+}^{\mathrm{PB}}(\lambda)$ curve, while the dotted thick line is for the $\kappa_{-}^{\mathrm{PB}}(\lambda)$ boundary. All of the region surrounded by these two curves corresponds to the PB region. We have also displayed the previously shown (in Fig. 1) $\kappa_{ \pm}^{\mathrm{NPB}}(\lambda)$ curves as dot-dashed thin and solid lines, respectively. The curves $\kappa_{\mathrm{QE}}(\lambda)$ and $\kappa=\lambda$ are shown as long-dashed thick and short-dashed thin lines, respectively.

$\kappa_{\mathrm{QE}}(\lambda)$ (in whose case the scaling variable is negative); or if on the contrary, the points are below this line, in whose case the scaling variable is positive.

The purpose of Fig. 4 is to highlight, in general, the smallness of the region of the $(\lambda, \kappa)$-space where PB plays a
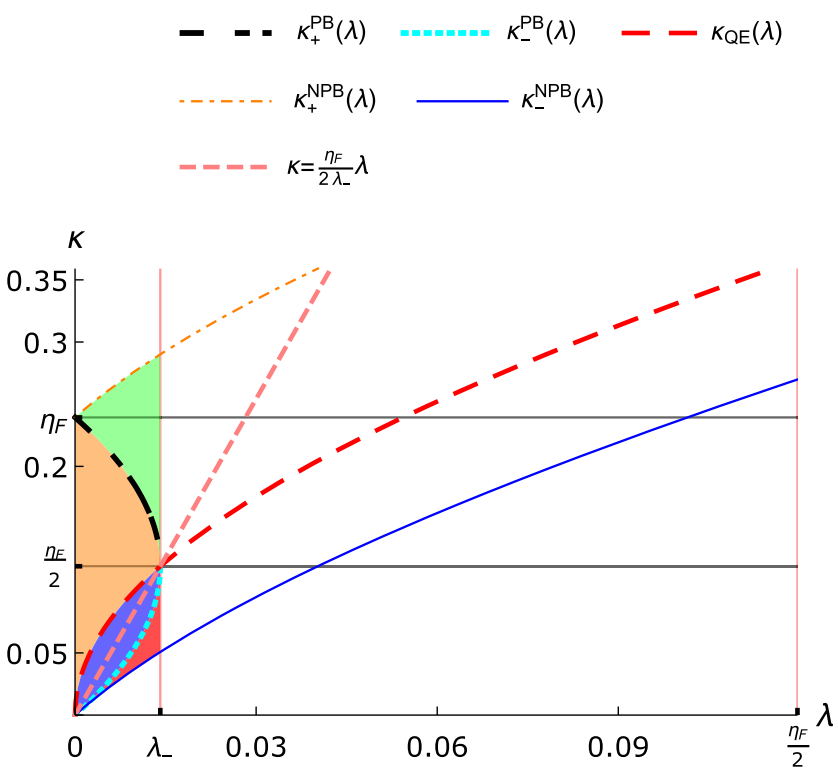

FIG. 4. Same plot as in Fig. 3, but highlighting the smallness of the region where PB plays a role. Notice that $\lambda_{-} \ll \eta_{F}$. Also shown a new straight line, $\kappa=\eta_{F} \lambda /\left(2 \lambda_{-}\right)$, in short-dashed style, that is entirely contained in the filled region between the longdashed and dotted curves, for the range of values $0 \leq \lambda \leq \lambda_{-}$. The purpose of this line and that corresponding to the horizontal line $\kappa=\eta_{F} / 2$ will be clear in Fig. 5 . 
role. Note that $\lambda_{-} \ll \eta_{F} / 2$ and that $\eta_{F}$ is not visible in the $\lambda$-axis due to units, while in the vertical axis it appears. Also notice that the horizontal straight line $\kappa=\eta_{F} / 2$ is entirely contained in the filled $\mathrm{PB}$ region above the curve of the $\mathrm{QE}$ peak (as far as $0 \leq \lambda \leq \lambda_{-}$), just as it happens for the $\kappa_{+}^{\mathrm{PB}}(\lambda)$ curve. The same can be said for the straight line $\kappa=\eta_{F} \lambda /\left(2 \lambda_{-}\right)$and the $\kappa_{-}^{\mathrm{PB}}(\lambda)$ curve in the filled $\mathrm{PB}$ region just below the QE peak position curve (long-dashed line). The purposes of these two straight lines will be clear in the following discussion.

In Fig. 5 we show the values taken by the scaling variable $\psi$ in the RFG model along different curves $\kappa=\kappa(\lambda)$ in the $(\lambda, \kappa)$-plane in the region where $0 \leq \lambda \leq \lambda_{-}$. Of course, we have shown the limiting boundaries $\psi= \pm 1$ given by the curves $\kappa_{\mp}^{\mathrm{NPB}}(\lambda)$, respectively. They correspond to the medium-thick solid and dot-dashed horizontal straight lines in Fig. 5, respectively; and to the curves of the same styles in Figs. 3 and 4. Along the long-dashed thick $\kappa_{\mathrm{QE}}(\lambda)$ curve of Figs. 3 and 4, the scaling variable is equal to zero in Fig. 5 because this is the curve where $\lambda=\tau$ and the sign function vanishes [see Eq. (6)].

The rest of curves shown, especially in Fig. 4, remains inside the $\mathrm{PB}$ region for $0 \leq \lambda \leq \lambda_{-}$. In this region, remarked by the filled region between $\kappa_{+}^{\mathrm{PB}}(\lambda)$ and $\kappa_{-}^{\mathrm{PB}}(\lambda)$ curves in Figs. 3 and 4, the scaling variable $\psi(\kappa(\lambda), \lambda)$ does not depend at all on the $\kappa$ value taken by any point or curve inside the region, except for the sign of $\psi$. This can be viewed in different forms. For instance, taking a look at the values taken by $\psi$ along the curves $\kappa_{+}^{\mathrm{PB}}(\lambda)$ (three-fold
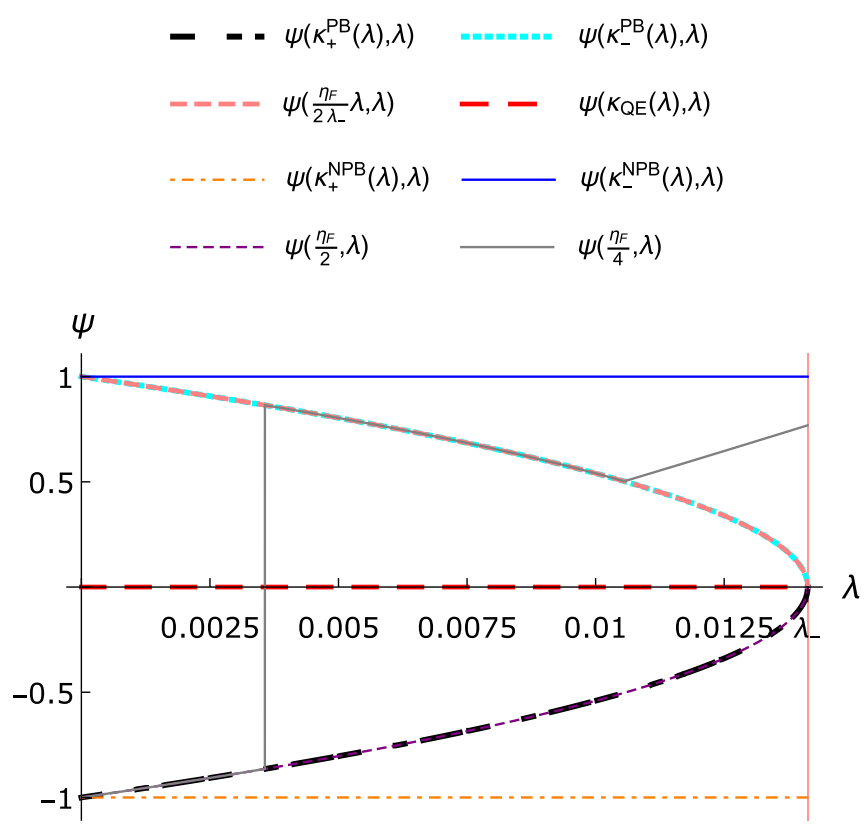

FIG. 5. Values taken by the scaling variable $\psi(\kappa(\lambda), \lambda)$ along the different curves shown in Figs. 3 and 4 in the PB region, i.e., when $0 \leq \lambda \leq \lambda_{-}$. For an exhaustive explanation see the main text. dashed thick line) and along the straight line $\kappa=\eta_{F} / 2$ (very short-dashed thin curve in Fig. 5): both curves are totally inside the filled PB region above the QE peak position curve of Figs. 3 and 4, however, their values of $\kappa$ along the curves are totally different, and still the scaling variable takes the same values in Fig. 5, i.e., it starts equaling $\psi=-1$ for $\lambda=0$ because then $\epsilon_{0}=\epsilon_{F}-2 \lambda=\epsilon_{F}$ and both curves are above the $\kappa=\kappa_{\mathrm{QE}}(\lambda)$ curve, thus having negative values for the scaling variable. Finally, for $\lambda=\lambda_{-}$, the scaling variable is zero along both paths because it is the intersection point with the $\kappa_{\mathrm{QE}}(\lambda)$ curve (see especially Fig. 3).

Something similar occurs along the paths defined by $\kappa_{-}^{\mathrm{PB}}(\lambda)$ (dotted thick line) and $\kappa=\eta_{F} \lambda /\left(2 \lambda_{-}\right)$(mediumdashed thick line), but in this case for positive values of the scaling variable, because in this case both paths are entirely in the filled PB region below the $\kappa_{\mathrm{QE}}(\lambda)$ curve of Fig. 4, thus in the region of positive values for the scaling variable, as it can be seen again in Fig. 5 .

The final example is a mixed case, a straight line $\kappa=\eta_{F} / 4$ (see Fig. 3) that starts in the filled PB region of negative values of the scaling variable $\psi$, passes across the $\kappa_{\mathrm{QE}}(\lambda)$ curve, enters in the filled $\mathrm{PB}$ region below the $\kappa_{\mathrm{QE}}(\lambda)$ line and, finally, it gets out of the PB region by entering entirely in the NPB region of positive values of $\psi$. In this case (corresponding to the solid thin line in Fig. 5), the initial behavior of the scaling variable is the same as that corresponding to the other curves lying entirely in the PB region above the $\kappa_{\mathrm{QE}}(\lambda)$ curve (negative values for $\psi$ ), until the point where $\kappa_{\mathrm{QE}}(\lambda)=\eta_{F} / 4$ (corresponding approximately to $\lambda \simeq 0.0036$ ), where the $\kappa=\eta_{F} / 4$ horizontal line enters in the $\mathrm{PB}$ region below the $\kappa_{\mathrm{QE}}(\lambda)$ curve, and it suddenly changes the sign of $\psi$ along this crossing point, as can be seen in Fig. 5 as the vertical solid thin line. Now the values of $\psi$ roam along those of any curve entirely contained in the PB region below the $\kappa_{\mathrm{QE}}(\lambda)$ curve (corresponding to positive values of $\psi$ ) until the new point where $\kappa_{-}^{\mathrm{PB}}(\lambda)=\eta_{F} / 4(\lambda \simeq 0.011)$, where the line $\kappa=\eta_{F} / 4$ enters finally in the NPB region. In this last region, however, $\epsilon_{0}$ is no longer equal to $\epsilon_{F}-2 \lambda$, but to $\kappa \sqrt{1+1 / \tau}-\lambda$, and then, while still having positive values, the scaling variable now approaches $\psi=+1$, which is what will happen when $\kappa_{-}^{\mathrm{NPB}}(\lambda)=\eta_{F} / 4$ (corresponding to a value of $\lambda \simeq 0.017>\lambda_{-}$, and therefore out of the range of Fig. 5).

\section{B. Analytical boundaries coming from the lepton kinematics}

Up to now, nothing has been imposed from the lepton kinematics, but we know that the final lepton scattering angle must be a physical one. We discuss in this section that imposing constraints from lepton kinematics (for a fixed initial neutrino/antineutrino energy) further restricts the available phase space for the RFG model; for a detailed 
derivation of some formulas relevant for this and future sections, the reader is referred to Appendix A 4. Here we only provide the relevant results.

The lepton kinematics' restrictions come from the allowed maximum and minimum final lepton energies (we will assume muon neutrinos, and so the final lepton will be a muon [65]) for a given initial neutrino/antineutrino energy,

$$
\begin{gathered}
\omega=E_{\nu}-E_{\mu} \Leftrightarrow E_{\mu}=E_{\nu}-2 m_{N} \lambda \\
q^{2}=\left(\vec{k}-\vec{k}^{\prime}\right)^{2}=E_{\nu}^{2}+k^{\prime 2}-2 E_{\nu} k^{\prime} \cos \theta_{\mu},
\end{gathered}
$$

where $E_{\nu}$ is the initial neutrino energy, $\theta_{\mu}$ is the muon scattering angle with respect to the direction of the incident neutrino, and $k^{\prime}=\sqrt{E_{\mu}^{2}-m_{\mu}^{2}}$ is the final muon momentum with energy $E_{\mu}$ and mass $m_{\mu}$.

The minimal muon energy is its mass and from this condition we can obtain from Eq. (10) the, in principle, maximum allowed value for $\lambda$,

$$
\lambda_{\max }=\frac{E_{\nu}-m_{\mu}}{2 m_{N}}=\epsilon_{\nu}-\tilde{m}_{\mu},
$$

where we have introduced "reduced" and dimensionless neutrino energy and muon mass variables, defined as

$$
\epsilon_{\nu} \equiv \frac{E_{\nu}}{2 m_{N}}, \quad \tilde{m}_{\mu} \equiv \frac{m_{\mu}}{2 m_{N}} .
$$

From Eq. (11) we can write that the absolute value of the cosine of the muon scattering angle must be lesser or equal to 1 :

$$
\begin{aligned}
\left|\cos \theta_{\mu}\right| \leq 1 & \Leftrightarrow\left|\frac{E_{\nu}^{2}+k^{\prime 2}-q^{2}}{2 E_{\nu} k^{\prime}}\right| \leq 1 \\
& \Leftrightarrow-2 E_{\nu} k^{\prime} \leq E_{\nu}^{2}+k^{\prime 2}-q^{2} \leq 2 E_{\nu} k^{\prime} .
\end{aligned}
$$

Notice that Eq. (13) gives two additional inequalities for $\kappa$ in terms of $\lambda$ [the variable $\lambda$ is hidden in $k^{\prime}$ via its dependence on $E_{\mu}$ and the dependence of the latter on $\lambda$ through Eq. (10)]. From the first inequality, and using the "reduced" and dimensionless variables, we obtain:

$$
\begin{aligned}
q^{2} & \leq\left(E_{\nu}+k^{\prime}\right)^{2} \\
& \Leftrightarrow \frac{q}{2 m_{N}} \leq \frac{E_{\nu}}{2 m_{N}}+\frac{\sqrt{\left(E_{\nu}-2 m_{N} \lambda\right)^{2}-m_{\mu}^{2}}}{2 m_{N}} \\
& \Leftrightarrow \kappa \leq \epsilon_{\nu}+\sqrt{\left(\epsilon_{\nu}-\lambda\right)^{2}-\tilde{m}_{\mu}^{2}} \equiv \kappa_{\max }^{\text {lepton }}(\lambda) .
\end{aligned}
$$

Analogously with the other inequality of expression (13), we obtain the lower bound for $\kappa$ constrained from the lepton kinematics alone:

$$
\kappa \geq \epsilon_{\nu}-\sqrt{\left(\epsilon_{\nu}-\lambda\right)^{2}-\tilde{m}_{\mu}^{2}} \equiv \kappa_{\min }^{\text {lepton }}(\lambda) .
$$

As the PB region [filled domains in Figs. 3 and 4 surrounded by the $\kappa_{+}^{\mathrm{PB}}(\lambda)$ and $\kappa_{-}^{\mathrm{PB}}(\lambda)$ curves] is always contained inside the larger region bounded by $\kappa_{-}^{\mathrm{NPB}}(\lambda) \leq$ $\kappa \leq \kappa_{+}^{\mathrm{NPB}}(\lambda)$, and the only difference between the PB region and the NPB one is the dependence of the scaling variable with $\kappa$ and $\lambda$; the furthest constrained phase space for the RFG model is given by

$$
\max \left(\kappa_{\min }^{\text {lepton }}, \kappa_{-}^{\mathrm{NPB}}\right) \leq \kappa \leq \min \left(\kappa_{\max }^{\text {lepton }}, \kappa_{+}^{\mathrm{NPB}}\right),
$$

provided that the maximum on the left-hand side of expression (16) is always smaller than the minimum on the right-hand side of the same expression in the range of $\lambda$-values ranging from $\lambda=0$ to $\lambda=\lambda_{\max }$, where $\lambda_{\max }$ is given in Eq. (12) for a fixed neutrino/antineutrino energy.

The curves $\kappa_{\max }^{\text {lepton }}(\lambda)$ and $\kappa_{\min }^{\text {lepton }}(\lambda)$, given in Eqs. (14) and (15), respectively, are monotonically decreasing and increasing with $\lambda$, respectively. Both curves reach the same value when $\lambda=\lambda_{\max }$, i.e., when $\kappa_{\max }^{\text {lepton }}\left(\lambda_{\max }\right)=$ $\kappa_{\min }^{\text {lepton }}\left(\lambda_{\max }\right)=\epsilon_{\nu}$.

In Fig. 6 we show the phase space in the $(\lambda, \kappa)$ variables for two different neutrino energies in the RFG model. We have also shown the cutting points between the different curves $\kappa_{\max , \operatorname{lin}}^{\text {lepto }}(\lambda)$ and $\kappa_{ \pm}^{\mathrm{NPB}}(\lambda)$, which constrain the lepton and nuclear model kinematics in the RFG, respectively. Note that, because we have shown the plots for the case of muon neutrinos, $m_{\mu}=106 \mathrm{MeV} / \mathrm{c}^{2}$, for neutrino energies close to the muon mass the phase space is mostly constrained by the lepton kinematics (left panel). However, for higher neutrino energies (right panel), the available phase space is almost entirely constrained by the nuclear model kinematics in the RFG (thin solid and dot-dashed lines corresponding to the limits of the RFG scaling function). In this latter case, lepton kinematics plays a really minor role, except in the region of the endpoint in $\lambda$, which corresponds to the largest energy transfers to the nucleus (and consequently the least energy carried by the muon), so one starts to see the effects of the muon mass as if one were in the situation of the left panel.

\section{SUSAM* MODEL CASE}

The $\mathrm{SuSAM}^{*}$ model is theoretically based in the Walecka (or $\sigma-\omega$ ) model $[66,67]$ for relativistic nuclear matter. The Walecka model was the first relativistic, manybody, quantum-field theory model that exhibited saturation in nuclear matter. The Relativistic Mean Field (RMF) version of the model for nuclear matter has constant scalar and timelike vector potentials, associated to the expectation values of the scalar and timelike component of the vector fields. However, for nuclear matter, the RMF version is exactly solvable and the dynamic nucleon fields can be 

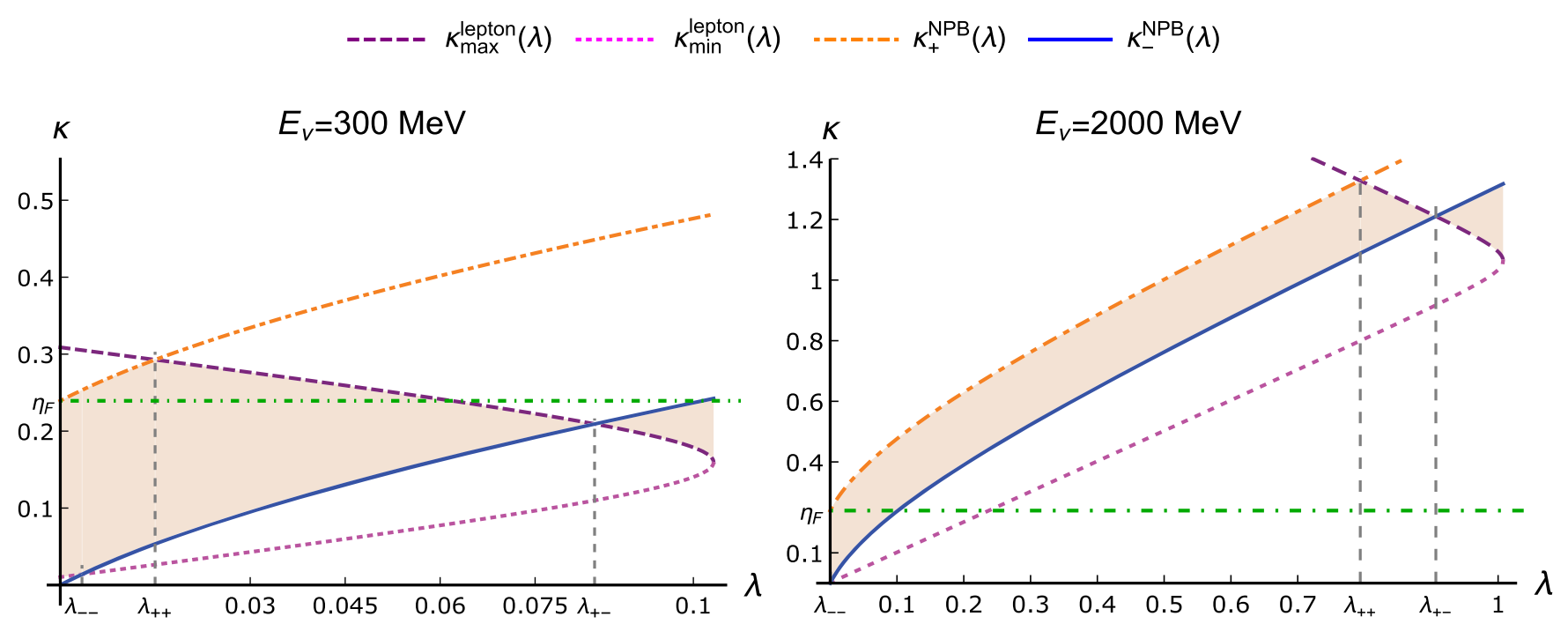

FIG. 6. Available phase space in $(\lambda, \kappa)$ variables in the RFG model for $E_{\nu}=300 \mathrm{MeV}$ (left panel) and for $E_{\nu}=2000 \mathrm{MeV}$ (right panel), shown as the shaded regions for $\lambda \leq \lambda_{+-}$. Also displayed are the different cut points between the curves constraining the lepton and nucleon kinematics, labeled as in Eqs. (A22) and (A24) of Appendix A 4. The value of $\eta_{F}$ has been taken as 0.239, corresponding to a Fermi momentum of $k_{F}=225 \mathrm{MeV} / \mathrm{c}$. Also note that the curves $\kappa_{\max \text {,min }}^{\text {lepton }}(\lambda)$ (dashed and dotted lines, respectively) are actually two different branches of the same curve.

expanded as plane-wave solutions as in a free theory, because the dynamics due to the scalar and vector potentials are hidden in a shift of the nucleon mass and the energy. Thus, the effect of the condensed value of the scalar field is to shift the mass of the nucleon, reducing it. What we have done in previous works [48-52] is to use this underlying well-founded theory to phenomenologically adjust the relativistic effective mass for several nuclear species, assuming that the "QE" electron scattering data scale within an uncertainty band, that has been also estimated; and that these "QE" electron scattering data can be selected from the whole inclusive data by means of a density criterion.

In this model, the Pauli blocking is treated exactly as in the RFG, i.e., by means of using Eq. (7). This treatment can be problematic and probably not excessively well founded, but it is the easiest way to incorporate it. For problems related to this treatment of the Pauli blocking in the SuSAM* model, the reader is referred to Sec. VA, where a more detailed discussion confronted with the results is given.

The binding energy in the SuSAM* model is simulated with the decrease in the nucleon mass, i.e., by using the relativistic effective (shifted by the condensed value of the scalar $\sigma$ field) nucleon mass, although this mass (and the Fermi momentum) is fitted for each nucleus for which we have inclusive $\left(e, e^{\prime}\right)$ data [52] from where selecting "QE" points using the density criterion mentioned above. This is not the first time such an attempt has been done to describe QE electron scattering (see also Refs. [68,69]), but, as far as we know, it is the first serious attempt to translate it to describe CCQE neutrino scattering.
In Fig. 7 we show the scaling functions of the two models we discuss in this article. In solid line style the scaling function of the RFG model is depicted, whose expression was given in Eq. (3). The other three scaling

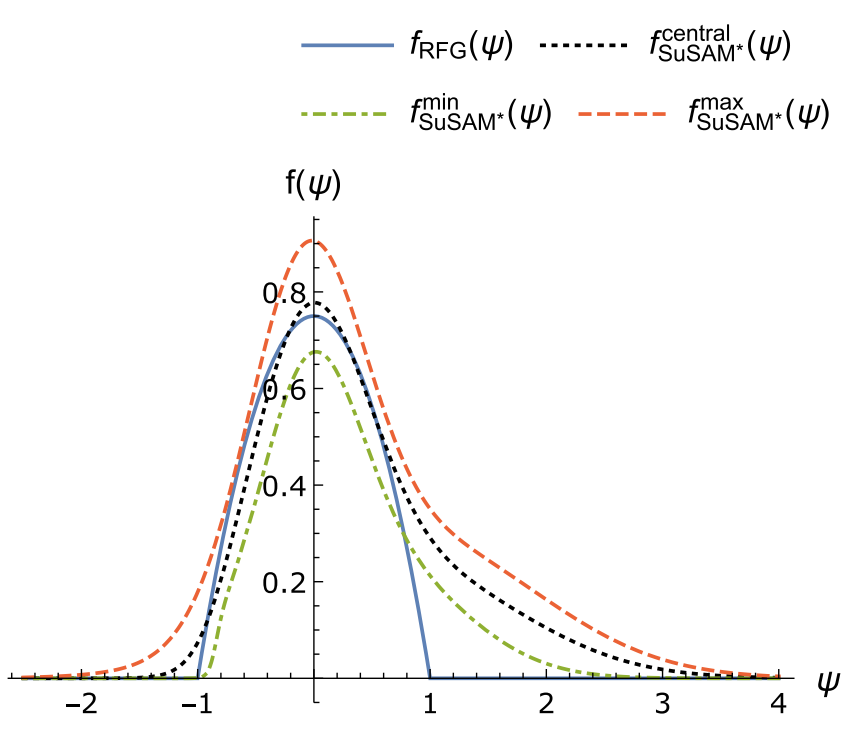

FIG. 7. Different scaling functions used in this article as a function of the scaling variable $\psi$. The well-known scaling function of the RFG model is shown in solid style, while the three SuSAM*-model scaling functions, extracted from a global fit to "QE" electron scattering data off nuclei in Ref. [52], are shown as dotted, dot-dashed, and dashed lines for the central, the lower and upper bounds, respectively. The scaling functions of the SuSAM* model plotted in this figure correspond to the parameters denoted as Band C in Table I of Ref. [52]. 
functions are those of the SuSAM* model, in particular, those extracted in a global fit to the world "QE" electron scattering data $[70,71]$ extracted out from the inclusive data stored in the web site of Ref. [70]. These three scaling functions (shown as central, min, and max in Fig. 7) were obtained in Ref. [52] after a selection procedure based on the scaling hypothesis of the QE data, and the lower and upper scaling functions correspond to the estimation of the uncertainty or thickness of the superscaling band where the bulk of the QE data tend to accumulate.

The functional form of the scaling functions of the SuSAM* model depicted in Fig. 7 is:

$$
f_{\text {SuSAM } *}(\psi)=\frac{a_{3} e^{-\frac{\left(\psi-a_{1}\right)^{2}}{2 a_{2}^{2}}}+b_{3} e^{-\frac{\left(\psi-b_{1}\right)^{2}}{2 b_{2}^{2}}}}{1+e^{-\frac{\psi-c_{1}}{c_{2}}}},
$$

where the parameters $a_{i}, b_{i}$, and $c_{i}$ can be found, for the three different scaling functions of Fig. 7, in Table I of Ref. [52], corresponding to the set labeled as Band C. It is worth noting the asymmetry shown by the scaling functions of the SuSAM* model, which have longer tails towards positive values of the scaling variable $\psi$ than they have for negative ones, in contrast with the symmetric RFG scaling function.

In Sec. III A we have discussed the boundaries in the $(\lambda, \kappa)$-plane where the scaling variable $\psi$ is between -1 and +1 , and therefore, this comprises the region where the RFG scaling function of Eq. (3) is different from zero. Because of this, the five response functions entering in the double differential (with respect to the final lepton kinematic variables) CCQE neutrino/antineutrino-nucleus cross section contribute only inside this boundary for the RFG model (see Secs. IIA and IIB of Ref. [51]).

Now, to do the same for the SuSAM* scaling functions, the procedure follows the lines sketched in Appendix A 2. In this appendix, we obtain $\kappa_{ \pm}^{\mathrm{NPB}}(\lambda)$ for the RFG model by imposing $\epsilon_{0} \equiv \kappa \sqrt{1+1 / \tau}-\lambda=\epsilon_{F}$, which is the equivalent condition to $\psi= \pm 1$. All we have to do to extend it for the $\mathrm{SuSAM}^{*}$ model is to identify extreme values of the scaling variable, namely $\psi_{\text {extr, }}$, where we can safely affirm that the SuSAM* scaling functions are negligible beyond these extreme values, one on the left and the other on the right. Note that, given the asymmetry of the SuSAM* scaling functions, these extreme values are not going to be necessarily the same at the left and at the right of the QE peak position.

Let us assume we are in the region where $\lambda>\tau$, and the sign function appearing in Eq. (6) is positive. If the positive extreme value $\left(\psi_{\text {extr }}\right)$ for the scaling variable in the SuSAM $^{*}$ model is larger than 1 , this obviously means that $\epsilon_{0}>\epsilon_{F}$. The limiting curve in the $(\lambda, \kappa)$ plane will be obtained when $\psi \equiv \sqrt{\frac{\epsilon_{0}-1}{\epsilon_{F}-1}}=\psi_{\text {extr }}$, where $\psi_{\text {extr }}$ has to be chosen properly as a large value where the scaling function of the SuSAM* model can be totally neglected beyond that value. This last equation is totally equivalent to:

$$
\epsilon_{0} \equiv \kappa \sqrt{1+\frac{1}{\tau}}-\lambda=1+\left(\epsilon_{F}-1\right) \psi_{\mathrm{extr}}^{2} .
$$

The same equation would have been obtained for the case $\lambda<\tau$, with the negative sign function in Eq. (6), as it also happened in the RFG case. Note that if we choose $\psi_{\text {extr }}= \pm 1$, we recover the condition of the RFG, $\epsilon_{0}=\epsilon_{F}$, as it should be.

To obtain the boundaries of the phase space in the $(\lambda, \kappa)$ plane for the SuSAM* model, note that Eq. (18) is the same as that for the RFG $\left(\epsilon_{0}=\epsilon_{F}\right)$ with the right-hand side replaced by $1+\left(\epsilon_{F}-1\right) \psi_{\text {extr }}^{2}$ instead of $\epsilon_{F}$. Consequently, we can take the Eq. (A11) of Appendix A 2 and replace any appearance of $\epsilon_{F}$ by the new $\epsilon_{F}^{\prime} \equiv 1+\left(\epsilon_{F}-1\right) \psi_{\text {extr }}^{2}$, where $\psi_{\text {extr }}$ does not necessarily have to be equal for the $\kappa_{+}^{\mathrm{NPB}}(\lambda)$ (corresponding to negative values of $\psi$ ) and for the $\kappa_{-}^{\mathrm{NPB}}(\lambda)$ (corresponding to positive values of the scaling variable) functions, because of the asymmetry of the scaling functions in the SuSAM* model.

The last important point that remains to be shown is that, for the SuSAM* model, it is still true that $\kappa_{-}^{\mathrm{NPB}}(0)=0$ and that $\kappa_{+}^{\mathrm{NPB}}(0)>\eta_{F} \equiv \sqrt{\epsilon_{F}^{2}-1}$ (these proofs are provided in Appendix B). This is important because mainly the effect of choosing a wider scaling function than that of the RFG is to broaden the available phase space shown in Fig. 6 between the thin dot-dashed and solid curves, thus increasing the domain of integration in $(\lambda, \kappa)$ space and obtaining a larger total cross section for a fixed neutrino/antineutrino energy, $\sigma\left(E_{\nu}\right)$.

The fact that $\kappa_{+}^{\mathrm{NPB}}(0)>\eta_{F}$ is related to the high momentum components, larger than the Fermi momentum, that real nuclei have in its ground state. These high momentum components, mainly produced by interaction and short-range correlations (SRC) [72-88], are totally missing in the RFG model, but not in the phenomenological scaling function of the SuSAM* model, which has been obtained from a global fit to selected "QE" electron scattering data from nuclei.

It is also well known that the effects of SRC are mainly present in the left tail of the scaling function [89-91], i.e., for $\psi_{\text {extr }}<-1$, which is precisely the left extreme $\psi$ value to be adequately chosen for the curve $\kappa_{+}^{\mathrm{NPB}}(\lambda)$, but they also show up in the right tail of the scaling function. This connection between high momentum components and scaling violations, and their effects in the total integrated QE neutrino cross section are deferred for a forthcoming study.

There are, in principle, other nuclear effects implicitly incorporated in the SuSAM* scaling function, which has been obtained in the scaling analysis of Ref. [52], by fitting globally all the inclusive $\left(e, e^{\prime}\right)$ scattering data of the nuclei present in the database of [70], but selecting only those 
"QE" points that scale within an uncertainty band, using a population density criterion to keep or reject them. Therefore, we expect that, besides SRC, other nuclear effects such as final-state interactions (FSI), long range correlations (RPA), 1p-1h and 2p-2h MEC contributions..., are also phenomenologically incorporated in the scaling function of the SuSAM* model.

However, a recent scaling re-analysis of the inclusive $\left(e, e^{\prime}\right)$ scattering data off ${ }^{12} \mathrm{C}$ has been carried out in Ref. [92], where the $2 \mathrm{p}-2 \mathrm{~h}$ MEC have been explicitly accounted for within the same model of RMF in nuclear matter with relativistic effective mass and vector energy in which the SuSAM* model is based. The authors have finally obtained essentially the same scaling function and band than in the SuSAM* model used here, even although the theoretical $2 \mathrm{p}-2 \mathrm{~h}$ MEC contribution was subtracted from the experimental data before carrying out the scaling analysis. These findings at least seem to hint that the $2 \mathrm{p}-2 \mathrm{~h}$ MEC contributions present in the electron scattering data are not so relevant to extract a $\mathrm{QE}$ scaling function.

In Fig. 8 we show the comparison between the available phase space in the RFG (already shown in Fig. 6) and SuSAM* scaling models, for the same two neutrino energies as in Fig. 6. The most remarkable difference is the enlargement of the phase space, shown as the paler shade, in the SuSAM* model. This enlargement is only attributable to the tails of the superscaling function of the SuSAM* model, which are absent in the RFG, because the curves delimiting the boundaries from the lepton kinematics constraints are the same, they do not depend at all on the scaling function. The main consequence of this enlargement of the phase space will be reflected in a larger total integrated cross section. Of course, this increase in the integrated cross section will depend on the values attained by the double differential [with respect to $(\lambda, \kappa)$ variables] CCQE cross section in the enlarged region. We can ensure that there is going to be a clear increase, because in the regions outside the phase space of the RFG, but close to its boundaries, the differential cross section will still be substantial because the scaling function of the SuSAM* is truly different from zero for scaling variables larger than 1 and lesser than -1 , which corresponds to points lying in the paler shaded regions. In any case, as expected, when the values of $(\lambda, \kappa)$ are approaching the two-fold thin dashed curve $\kappa_{+\mathrm{SuSAM} *}^{\mathrm{NPB}}(\lambda)$ and the long-dashed thin line $\kappa_{-\mathrm{SuSAM} *}^{\mathrm{NPB}}(\lambda)$, their contribution to the total cross section will be very small because in these zones of the phase space the SuSAM* scaling function becomes negligible.

It is also worth noting that the SuSAM* boundaries have been calculated in Fig. 8 for $\psi_{\text {left }}=-2.5$ and $\psi_{\text {right }}=6$ for the upper and lower SuSAM* boundaries, corresponding to the two-fold dashed and long-dashed thin curves, respectively. These extreme values of the scaling variable for the left and right tails of the SuSAM* superscaling function have been chosen thinking in the values attained by the central SuSAM* function (shown in Fig. 7) at them, which amount to roughly a factor $10^{-7}$ of the value of this scaling function at the peak. Also, in Fig. 8 we show, in the $\kappa$-axis, the value $\eta_{F}^{\prime} \approx 0.62$, defined as

$$
\eta_{F}^{\prime}=\sqrt{\epsilon_{F}^{\prime 2}-1},
$$

with $\epsilon_{F}^{\prime}=1+\left(\epsilon_{F}-1\right) \psi_{\text {left }}^{2}$ as the horizontal thin dotdashed line.
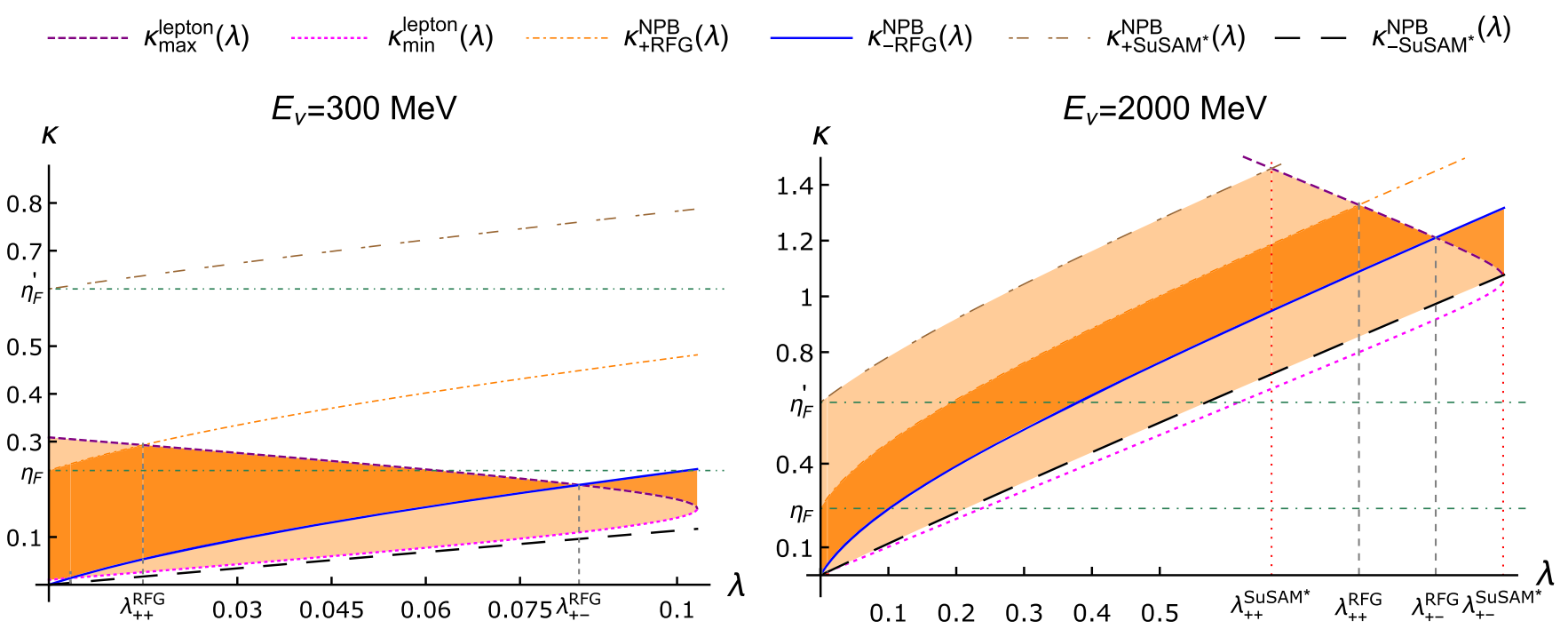

FIG. 8. Comparison of the available phase spaces in $(\lambda, \kappa)$ variables in the RFG (darker shade) and SuSAM* (paler shade) models for $E_{\nu}=300 \mathrm{MeV}$ (left panel) and for $E_{\nu}=2000 \mathrm{MeV}$ (right panel), shown as the shaded regions for $\lambda \leq \lambda_{+-}^{\text {model }}$, in general. Also displayed are the different cut points between the curves constraining the lepton and scaling model kinematics, labeled as in Fig. 6 for the different models. Note that the available phase space gets much more enlarged in the SuSAM* model, due to the tails of the scaling function. 
The analytical delimitation of the phase space boundaries in the $(\lambda, \kappa)$ variables is important because when integrating over the final lepton kinematics in order to obtain the total CCQE integrated cross section as a function of the neutrino/antineutrino energy, we can make the integration procedure as efficient as possible, as we are evaluating the integrand only where it is different from zero. This is particularly important when the neutrino energy is really huge, $E_{\nu} \sim 50-100 \mathrm{GeV}$, because then the contribution of the QE peak is concentrated at small values of the energy transfer $\omega$ (if compared with the neutrino energy) and at very forward angles. As for huge neutrino energies the allowed interval in $\omega$ is also huge, it is convenient to constrain as much as possible the angular interval (related to $\kappa$ ) where truly integrating.

\section{RESULTS}

In this section, we show the results for the CCQE double differential $\frac{d^{2} \sigma}{d T_{\mu} d \cos \theta_{\mu}}$ and $\frac{d^{2} \sigma}{d \kappa d \lambda}$ neutrino and antineutrino cross sections for the RFG and SuSAM* models, as well as the fully integrated total cross sections in both models.

\section{A. Double differential cross sections}

In Fig. 9 we show the double differential CCQE $\frac{d^{2} \sigma}{d \kappa d \lambda}$ cross section per neutron for the $\left(\nu_{\mu}, \mu^{-}\right)$reaction off ${ }^{12} \mathrm{C}$, at incident neutrino energies of $300 \mathrm{MeV}$, for the two models discussed in this work: RFG (left panel) and SuSAM* (right panel). The available phase spaces in the two models at this neutrino energy are those already depicted in the left panel of Fig. 8. Note that, although not exactly the same, both scales in the two panels are very similar, as well as the values reached by the cross section. In Fig. 9 we also show as the short-dashed line the curve $\kappa=\kappa_{\mathrm{QE}}(\lambda)$, where $\lambda=\tau$ and $\psi=0$, i.e., the curve corresponding to the position of the QE peak, which, as expected, runs over the region of largest cross section. In solid style, it is also shown the boundary of the $\mathrm{PB}$ region. As already mentioned in Sec. III A, in the PB region the scaling variable $\psi$ only depends on $\lambda$ and not on $\kappa$. Because of this, the contour lines (curves with the same value of the cross section) inside the PB region are almost vertical lines, because the dependence on $\kappa$ mainly enters through the lepton kinematic factors $V_{K}$ and the nuclear response functions $U_{K}$ of Eq. (2) and it is very mild at least for the RFG (left panel) model. Notice also that at the boundaries of the PB region, the contour lines show a sudden change of their direction. This is because at these boundaries the scaling variable $\psi$ starts to sharply depend on $\kappa$ as well.

There is, nevertheless, a remarkable difference between the left and right panels of Fig. 9 in the PB region: in the SuSAM* model (right panel) the color gradient along vertical lines of constant $\lambda$ changes abruptly when crossing the QE curve, especially for small values of $\lambda$; however this effect is totally absent in the left panel, corresponding to the RFG model. The reason for this is because of the properties of the scaling functions in the two models. In the RFG, the scaling function given by Eq. (3) is an even function of $\psi$. This means that for constant $\lambda$ there is no difference in being above or below the $\mathrm{QE}$ curve inside the $\mathrm{PB}$ region (the only difference is the sign of the scaling variable, but not the value of the scaling function in the RFG model). However, the situation is very different in the SuSAM*
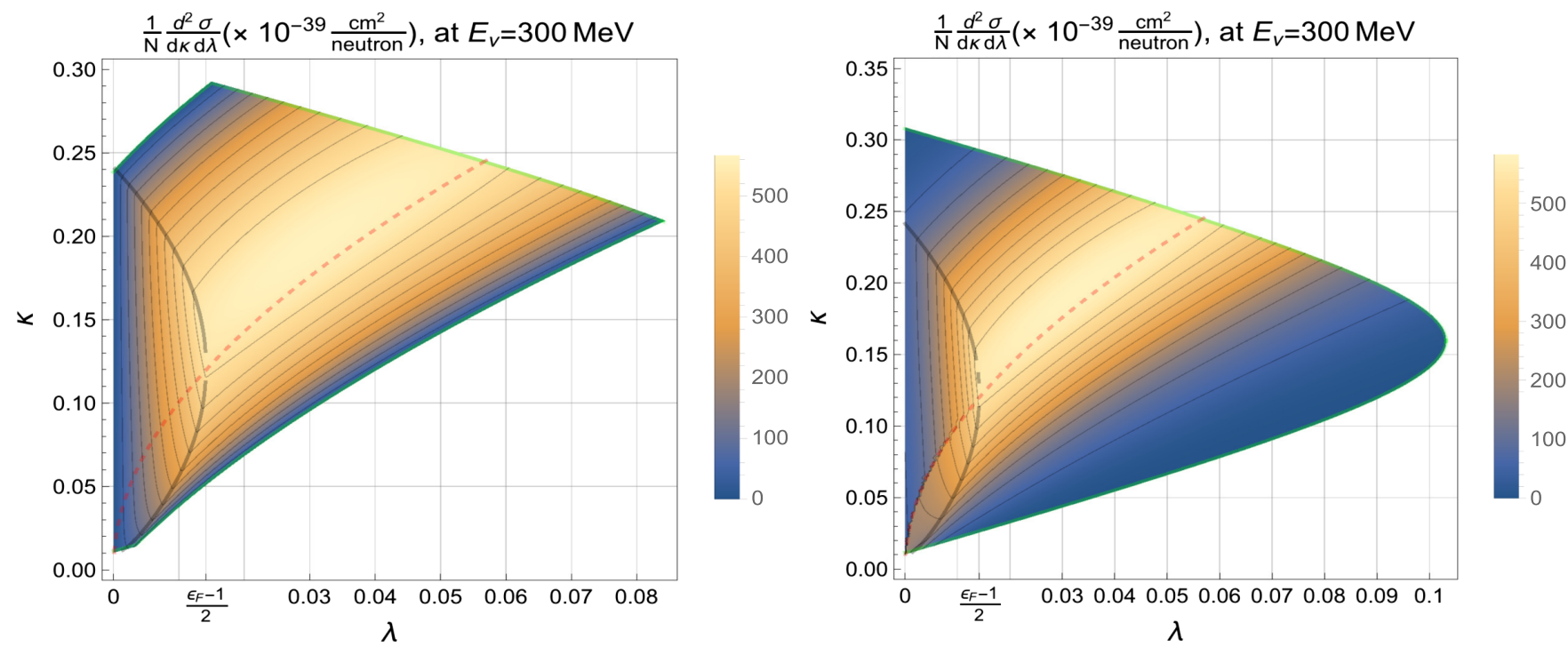

FIG. 9. Comparison of the density plots for the $\nu_{\mu}$ CCQE double differential $\frac{d^{2} \sigma}{d \kappa d \lambda}$ cross section per neutron in ${ }^{12} \mathrm{C}$ for the RFG (left panel) and SuSAM* (right panel) models at $E_{\nu}=300 \mathrm{MeV}$. Note that the available phase spaces in the different models are those shown in the left panel of Fig. 8. We show in short-dashed style the curve $\kappa_{\mathrm{QE}}(\lambda)$, where the QE peak is placed; while in solid fashion we also display the boundary of the PB region, already shown in Figs. 3 and 4. 

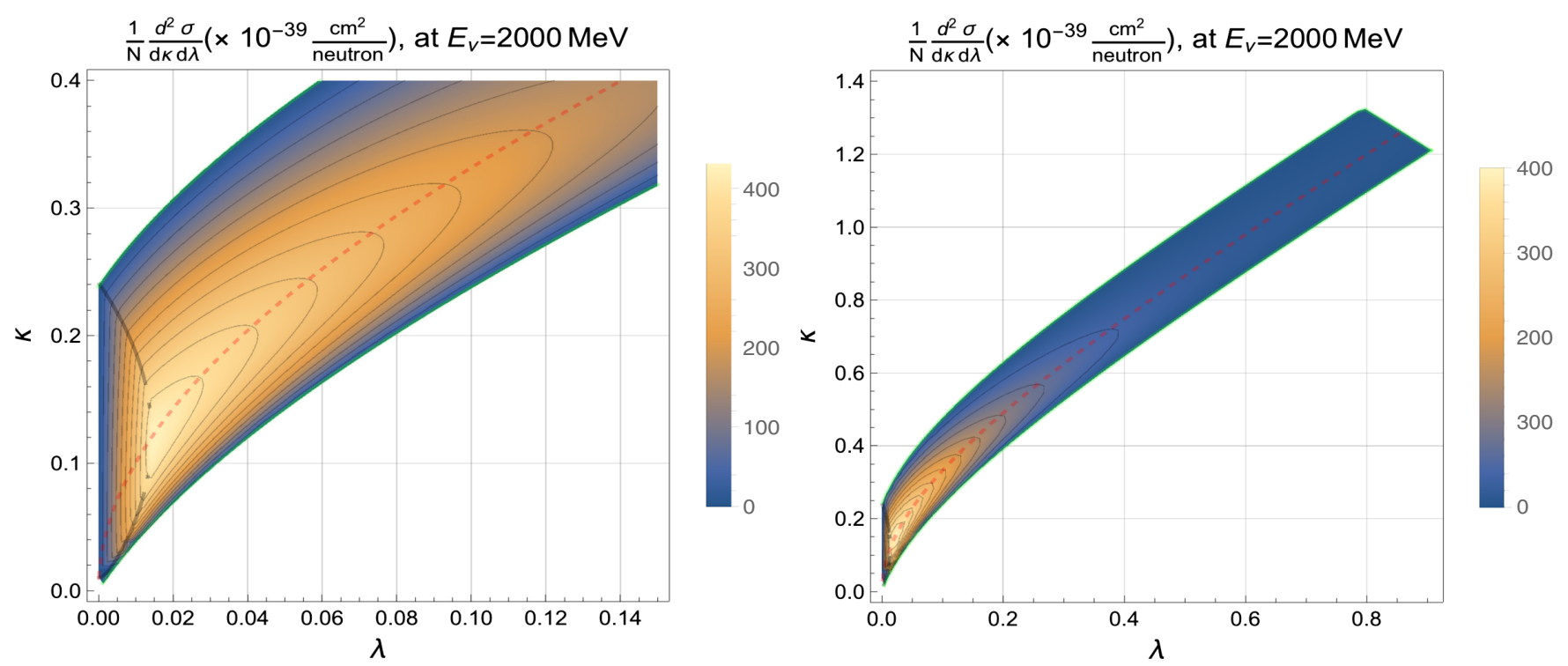

FIG. 10. Density plots for the $\nu_{\mu}$ double differential CCQE $\frac{d^{2} \sigma}{d \kappa d \lambda}$ cross section per neutron in ${ }^{12} \mathrm{C}$ in the RFG model at $E_{\nu}=2000 \mathrm{MeV}$. The left panel highlights the PB region, while the right one shows the full phase space region corresponding to those already shown in the right panels of Figs. 6 and 8. Lines have the same meaning as in Fig. 9.

model because its scaling function, given by Eq. (17), also depends on $\psi$ and not only on $\psi^{2}$. Hence, a simple change of sign in the scaling variable can produce a large difference in the scaling function (see dotted line of Fig. 7), thus inducing a sudden change in the value of the cross section when passing from positive values of the scaling variable (below the short-dashed curve) to negative ones (above the same curve) in the PB region. Nonetheless, this effect seems to be quite pronounced only for small values of $\lambda$ in the $\mathrm{PB}$ region, and not so perceptible for $\lambda$ values closer to the end point of the PB region, given by $\lambda_{-}$in Eq. (A21).

The physical reason for this sharp discontinuity in the value of the differential cross section when crossing the $\kappa_{\mathrm{QE}}(\lambda)$ curve inside the PB region can be surely related to the treatment of the Pauli blocking effect in the SuSAM* model. As discussed already in Sec. IV, the treatment of the Pauli blocking in the SuSAM* model is exactly the same as that of the RFG, which for sure is not the ideal one, although being the simplest one. For instance, in Refs. [64,93] for the SuSA and SuSAv2 models, a totally different and surely more well-founded approach has been used to incorporate the Pauli blocking, the so-called "mirror" scaling function subtraction. At the present stage of the SuSAM* model we have treated the Pauli blocking in the simplest way, and although we are aware of this limitation (that could be amended in future refinements of the model), it is not the purpose of this article to discuss these drawbacks in detail. The fairest option we can take is to warn the reader of this issue and of other possible ways of incorporating the Pauli blocking when using "by hand" phenomenological scaling functions that do not derive from a well-known momentum distribution, as it is also the case of the SuSAM* model.
In Figs. 10 and 11 we show the density and contour plots of the double differential CCQE $\frac{d^{2} \sigma}{d k d \lambda}$ cross section per neutron for $\nu_{\mu}$ reactions on ${ }^{12} \mathrm{C}$ at a fixed neutrino energy of $2000 \mathrm{MeV}$. Figure 10 corresponds to the RFG model, while Fig. 11 shows the results for the SuSAM* one. Left panels highlight the region of small values of energy transfers $\lambda$, i.e., showing clearly the PB region, while right panels in both figures show the full phase space. At this neutrino energy, the boundary of the phase space is basically delimited by the curves obtained from the scaling model conditions (limited by imposing the condition that the scaling function is zero or negligible), and not from the lepton kinematics, as it happened in Fig. 9 for smaller neutrino energy. Besides that, the sharp boundaries of the phase space for the RFG model, shown in Fig. 10, are due to the sharp way in which the RFG scaling function goes to zero at $\psi= \pm 1$. However, in Fig. 11, the phase space extends further than for the RFG case just because the SuSAM* scaling function has tails beyond $\psi= \pm 1$. Actually, the phase space of the SuSAM* model would extend even further than what is shown in Fig. 11, but with negligible values of the cross section, already visible in the own figure.

In general, the values of the cross section in both models at $E_{\nu}=2000 \mathrm{MeV}$ are very similar in the same regions of the $(\lambda, \kappa)$ phase space. In Fig. 11, there seems to be a nonnegligible cross section in the SuSAM* model in regions of the phase space below and close to the lower boundary of the RFG model, according to its color legend. These additional contributions will have a large impact in the total integrated $\mathrm{CCQE}$ cross section shown later on in Fig. 16 at $E_{\nu}=2000 \mathrm{MeV}$. 

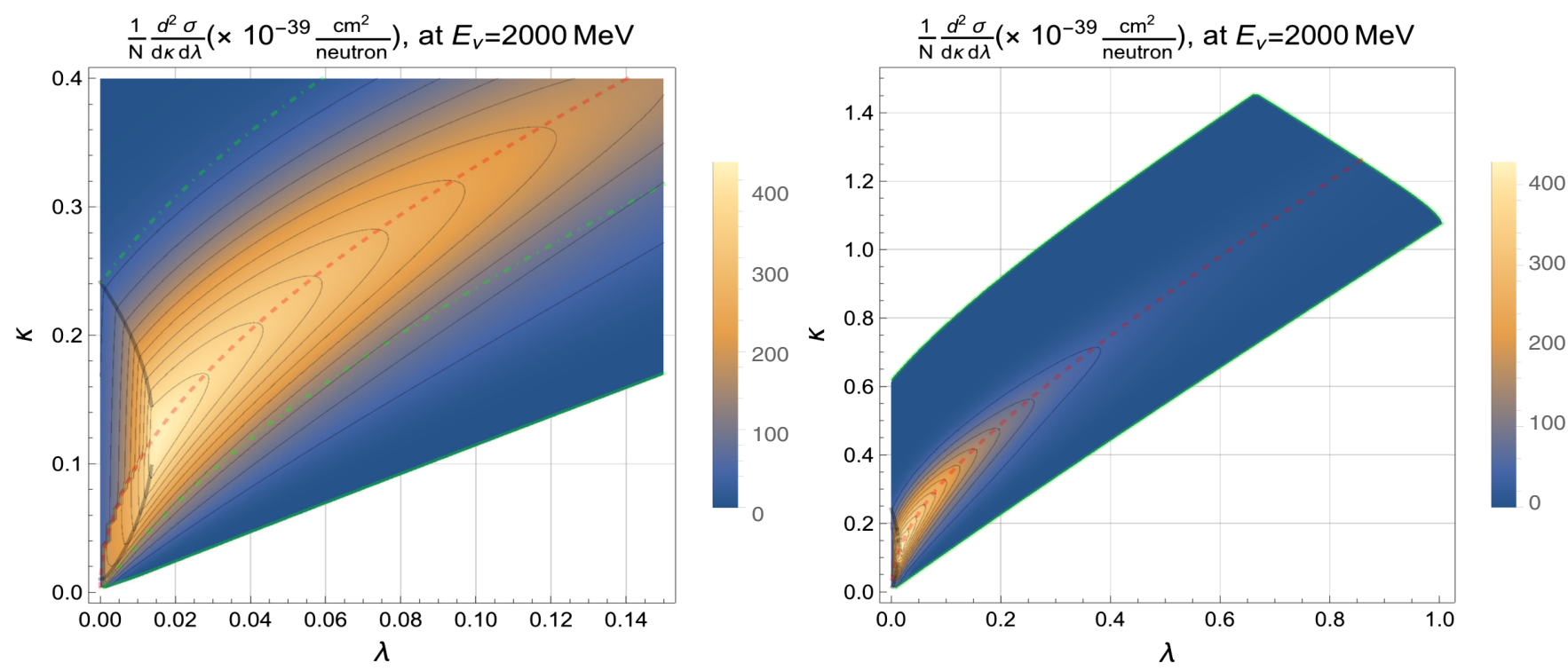

FIG. 11. Same as Fig. 10 but for the SuSAM* model. Short-dashed and solid lines have the same meaning as in Figs. 9 and 10. Notice, however, in the left panel, the dot-dashed lines that correspond to the upper and lower boundaries of the RFG model, i.e., the same boundaries shown in the left panel of Fig. 10.

Nonetheless, the most important feature of Figs. 10 and 11, if compared with Fig. 9 for $E_{\nu}=300 \mathrm{MeV}$ of incident neutrino energy, is that the maximum of the double differential cross section $\frac{d^{2} \sigma}{d \kappa d \lambda}$ depends very little on the neutrino energy. Indeed, for $E_{\nu}=300 \mathrm{MeV}$ the maximum of the cross section is around $550 \times 10^{-39} \mathrm{~cm}^{2} /$ neutron, while for $E_{\nu}=2000 \mathrm{MeV}$ this maximum is around $450 \times 10^{-39} \mathrm{~cm}^{2} /$ neutron. This remarkable feature makes this double differential cross section especially well suited to be used in $\mathrm{MC}$ generators to select the kinematics of the final lepton events for fixed neutrino energy. This is especially relevant for the generators that use the acceptance-rejection method to select the events, because using this method it is necessary to normalize the double differential cross section to its maximum value. And if this maximum value depends very weakly with the neutrino energy, one can efficiently set a fixed maximum suitable for all the neutrino energies. We will see that this efficiency would not be so attainable if one uses the double differential cross section $\frac{d^{2} \sigma}{d T_{\mu} d \cos \theta_{\mu}}$, given in Eq. (2), instead of $\frac{d^{2} \sigma}{d \kappa d \lambda}$, just because the maximum of the former depends very strongly on the neutrino energy.

Indeed, if we inspect Fig. 12, where the CCQE $\nu_{\mu}$-double differential cross section $\frac{d^{2} \sigma}{d T_{\mu} d \cos \theta_{\mu}}$ per neutron has been plotted for three different neutrino energies in the SuSAM* model, we can conclude two main things: First, the height of the peak of this cross section is strongly growing with the neutrino energy, as it can be seen from the values taken in the graduated color scale. Second, the larger the neutrino energy is, the more concentrated the bulk of the cross section is in a smaller region of the phase space, although this last conclusion can get overshadowed by the differences in the figures' scales. Moreover, the gradient of the cross section grows strongly with the neutrino energy for this differential cross section (larger variations of the cross section in a smaller region of the phase space, which makes the contour lines of constant cross section to appear closer and closer as the neutrino energy increases). Note, in particular, that this behavior is very striking in the bottom panel of Fig. 12, i.e., for $E_{\nu}=20 \mathrm{GeV}$. In this latter panel, in the bottom left corner, there is a white hole which means that there, the double differential cross section is much larger than the maximum value shown in its scale.

These conclusions should be compared with those of the $\frac{d^{2} \sigma}{d \kappa d \lambda}$ cross section per neutron shown in Figs. 9-11, where they were the opposite, i.e., the peak of the cross section was almost flat with the neutrino energy (we have also checked that this statement is also true for $E_{\nu}=20 \mathrm{GeV}$, although not shown in any figure), and the variation of the cross section over the phase space is much softer. These two special features make the double differential cross section $\frac{d^{2} \sigma}{d \kappa d \lambda}$ much more suitable to generate the final lepton events in any MC generator, specially those which use the acceptance-rejection method. Indeed, the event generation consists in the following steps:

(i) randomly select a point in kinematic phase space, e.g., $\lambda_{0}$ and $\kappa_{0}$;

(ii) randomly choose uniformly distributed variable $t$ in range $(0,1)$

(iii) accept event if $\left.\frac{d^{2} \sigma}{d \kappa d \lambda}\right|_{\lambda=\lambda_{0}, \kappa=\kappa_{0}}>t \max \left(\frac{d^{2} \sigma}{d \kappa d \lambda}\right)$ and reject otherwise.

For this method to be efficient, the differential cross section has to be as flat as possible for all neutrino energies 


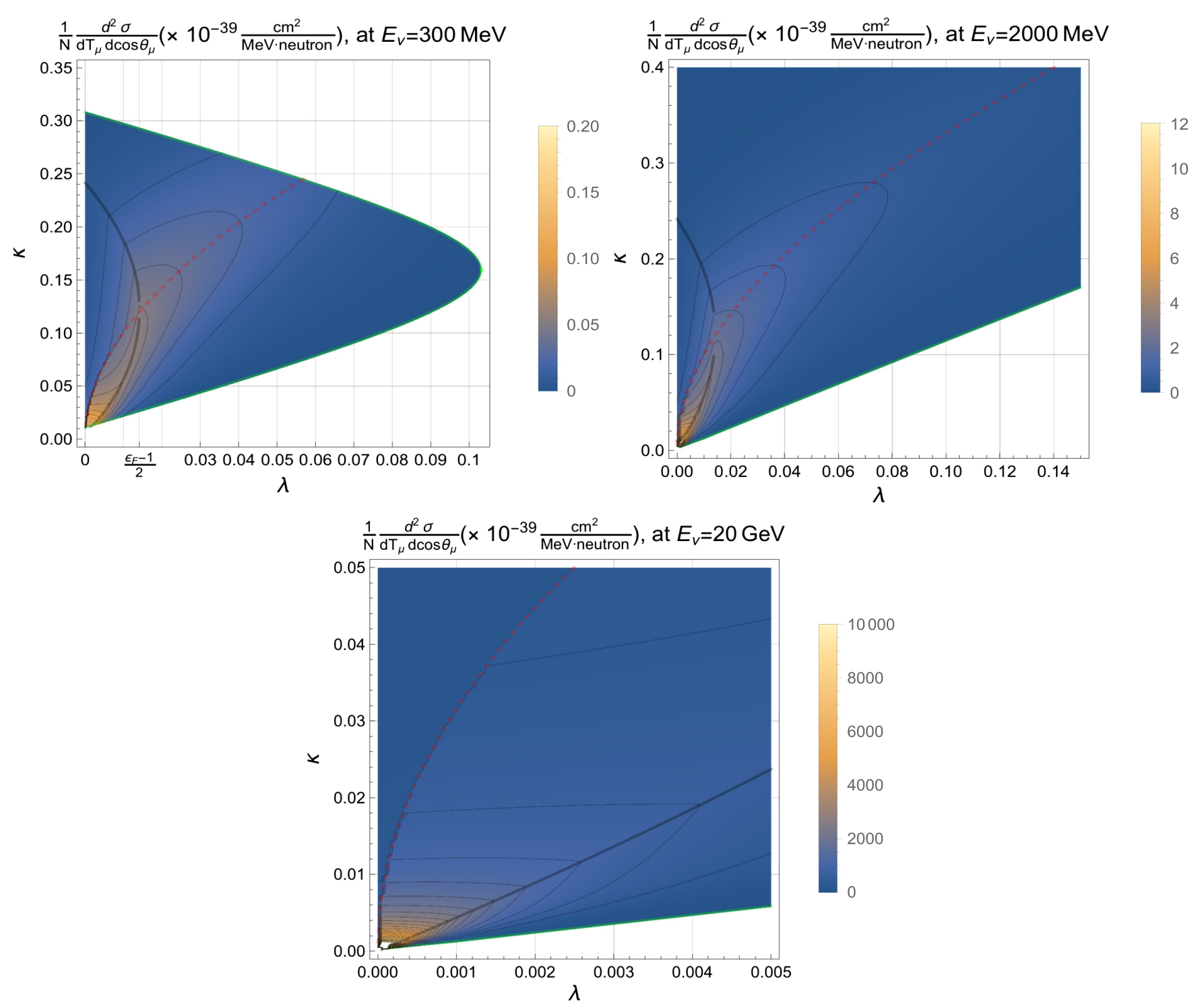

FIG. 12. Comparison of the density plots for the CCQE $\nu_{\mu}$-double differential cross section $\frac{1}{N} \frac{d^{2} \sigma}{d T_{\mu} d \cos \theta_{\mu}}$ off ${ }^{12} \mathrm{C}$ in the SuSAM* model for three different neutrino energies, highlighting the PB region, where the bulk of the cross section is concentrated. In the top left panel we show the density plot for $E_{\nu}=300 \mathrm{MeV}$; in the top right panel we display that for $E_{\nu}=2000 \mathrm{MeV}$; while in the bottom panel the plot for $E_{\nu}=20 \mathrm{GeV}$ is shown as well. Curves on the plot have the same meaning as they had in Figs. 9-11. The white hole in the bottom left corner of the bottom panel means that in that region the cross section is reaching values larger than the maximum shown in its scale.

because of two main reasons: first, it allows the maximum search algorithm to be more efficient (seeking the maximum more accurately in a shorter time); second, the fewer attempts to select kinematic variables that are rejected, the faster the events are generated.

In Figs. 13 and 14 we show the CCQE $\bar{\nu}_{\mu}$-induced double differential cross section $\frac{d^{2} \sigma}{d \kappa d \lambda}$ per proton for two different antineutrino energies, respectively. In the left panels we display the density plots for the RFG model, while in the right ones we show those for the SuSAM* model. The main conclusion that can be drawn from these figures if compared with the corresponding ones for the neutrino case is that, as expected, the antineutrino cross sections are smaller than their neutrino counterparts. This is especially clear in Fig. 13, if compared with Fig. 9, because the values in the scales of the figure for antineutrinos are roughly half of the values shown in Fig. 9, and the regions where the maximum values are reached in Fig. 13 are clearly smaller in size than those of Fig. 9, despite the fact that the available phase space is exactly the same.

The comparison can be less clear for the case of antineutrinos of $E_{\bar{\nu}}=2000 \mathrm{MeV}$ (Fig. 14) if one compares the corresponding model with the left panels of Figs. 10 and 11 , because in this case the color scales reach similar 

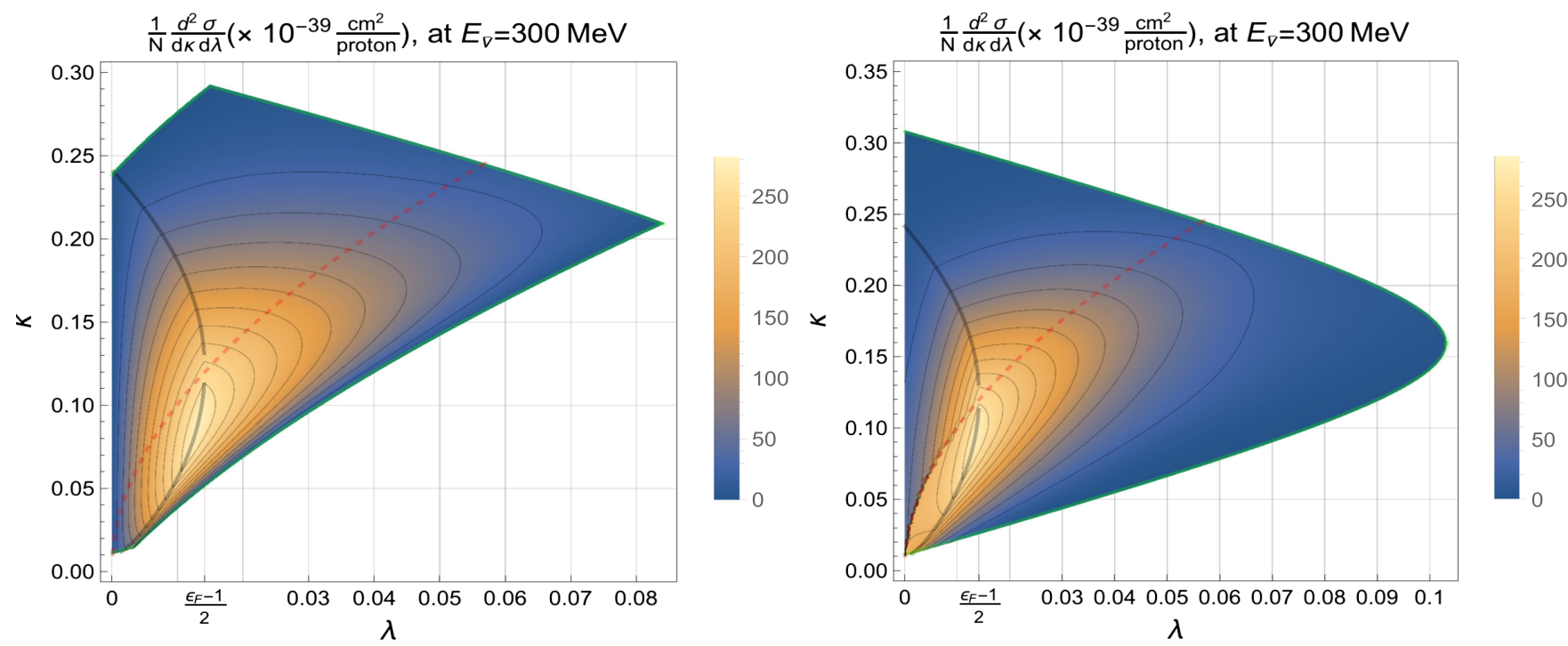

FIG. 13. Comparison of the density and contour plots for the $\bar{\nu}_{\mu} \mathrm{CCQE}$ double differential $\frac{d^{2} \sigma}{d \kappa d \lambda}$ cross section per proton in ${ }^{12} \mathrm{C}$ for the RFG (left panel) and SuSAM* (right panel) models at $E_{\bar{\nu}}=300 \mathrm{MeV}$. Lines have the same meaning as in Fig. 9. Note that now the cross section is roughly half than that for the neutrino case, but also much smaller along other regions of the whole phase space because of the minus sign in Eq. (2), which applies for CCQE antineutrino scattering. Notice as well about the difference this minus sign makes in the contour lines of constant double differential cross section.

values, although a bit smaller for antineutrinos. However, one can notice that the number of contour lines of constant cross section that enter completely inside the shown phase space (this is the same area of phase space shown in the left panels of Figs. 10 and 11) is larger in Fig. 14 (8 contour lines out of 10) than it was for the neutrino case ( 6 contour lines out of 10). This means that, even although the color scales could be considered similar, the contour lines for the antineutrino case appear more concentrated in the same region of phase space than their neutrino counterparts. Thus, we can conclude that larger cross sections extend far beyond the same phase space shown in Fig. 14 for the neutrino case than for the antineutrino one, yielding a larger CCQE total cross section for neutrinos than for antineutrinos.
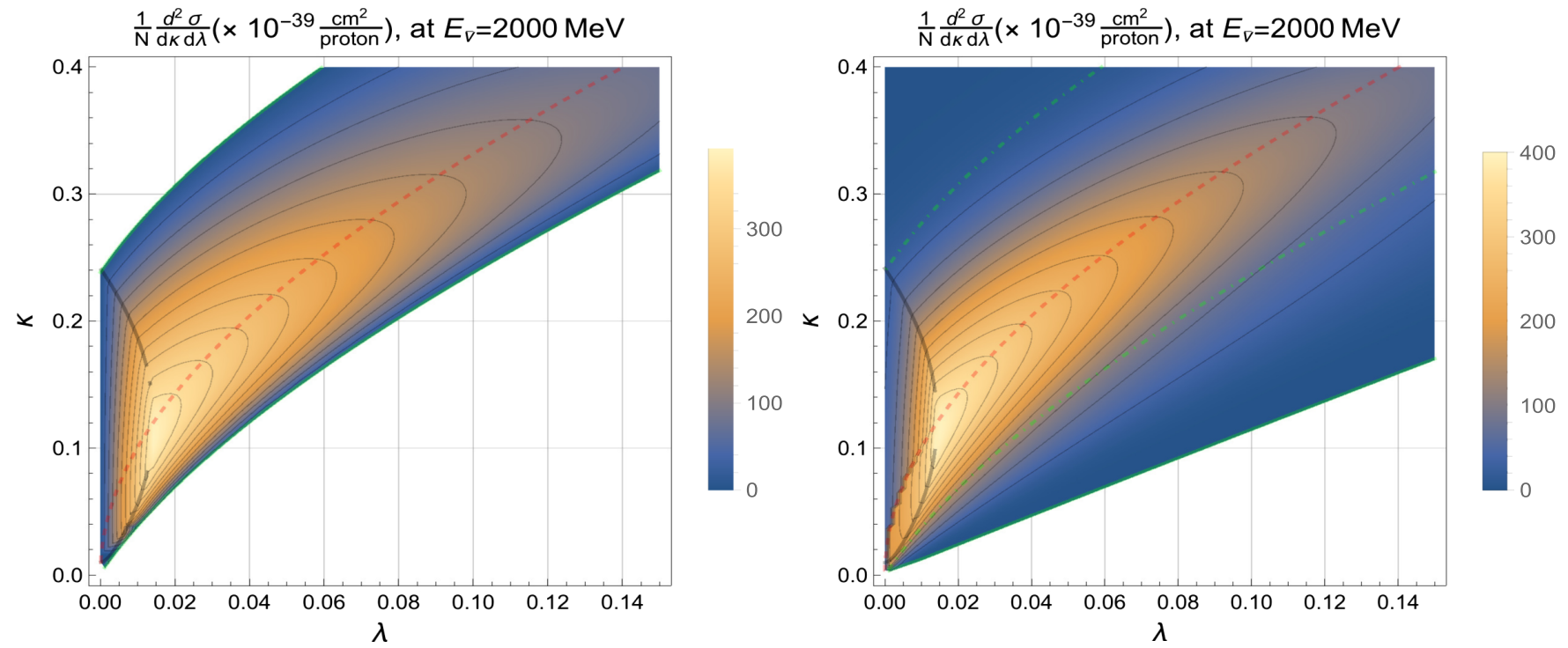

FIG. 14. Same as Fig. 13 but for $E_{\bar{\nu}}=2000 \mathrm{MeV}$. The left panel (RFG model) should be compared with the left panel of Fig. 10 , while the right one (SuSAM* model) should be compared with the left one of Fig. 11. These plots highlight the PB region and do not show the full phase space. Note again that the double differential cross section is smaller for CCQE antineutrino scattering than it is for neutrino case. The dot-dashed lines in the right panel are the boundaries of the RFG model shown in the left panel. 


\section{B. Total integrated cross section}

In this section we discuss the integrated CCQE cross sections both for neutrinos and antineutrinos off ${ }^{12} \mathrm{C}$, when integration over the $(\lambda, \kappa)$ phase space is carried out.

First of all, we want to point out a thorough description of how the $(\lambda, \kappa)$ phase space behaves as the neutrino energy increases. Notice that the curves $\kappa_{ \pm}^{\mathrm{NPB}}(\lambda)$, either those described by Eq. (9), or by Eq. (A11) (which are actually the same expressions, as explained in Appendix A 2), do not depend at all on the reduced neutrino energy $\epsilon_{\nu}$. Therefore these boundaries are always the same irrespective of the values taken by the neutrino energy. The dependence on the neutrino energy is in the curves $\kappa_{\max , \min }^{\text {lepton }}(\lambda)$ given by Eqs. (14) and (15).

At low neutrino energies, the phase space is completely bounded by the final lepton kinematics, i.e., by the curves $\kappa_{\max , \min }^{\text {lepton }}(\lambda)$ solely. This is the case, for instance, of the right panels of Figs. 9 and 13. In this case, there are no cutting points between the two lepton kinematic branches and between the upper and lower $\kappa_{ \pm}^{\mathrm{NPB}}(\lambda)$ curves. This same effect would occur in the left panels of Figs. 9 and 13, corresponding to the RFG model, but at a neutrino energy lower than $300 \mathrm{MeV}$, because in the RFG the $\kappa_{ \pm}^{\mathrm{NPB}}(\lambda)$ curves are squeezed with respect to those of the SuSAM* model. This can be seen, for instance, in the top left panel of Fig. 15 for the RFG.

As the neutrino energy increases, the cutting points between the curves $\kappa_{\max , \min }^{\text {lepton }}(\lambda)$ and $\kappa_{ \pm}^{\mathrm{NPB}}(\lambda)$ start to appear. One of these cuts is discussed in Appendix A 4, labeled as $\lambda_{++}$, given in Eq. (A22) and shown in Figs. 6 and 8. The other two additional cuts that can occur are those given by Eq. (A24), labeled as $\lambda_{ \pm-}$, and shown in Figs. 6 and 8 as
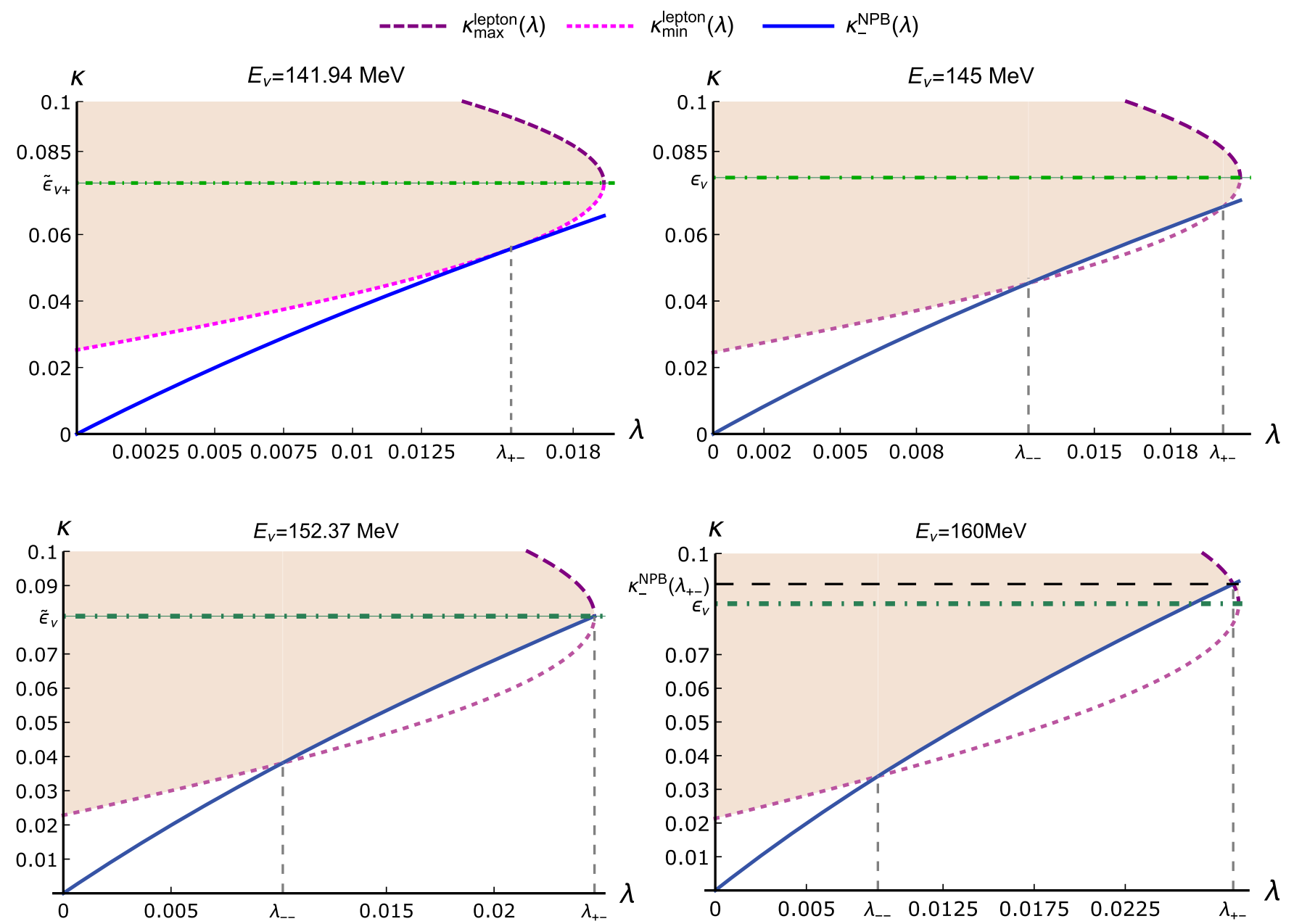

FIG. 15. Plot of the phase space for the RFG model at four different neutrino energies, where different situations arise. In the top left panel, the reduced neutrino energy is given by $\tilde{\epsilon}_{\nu+}$ in Eq. (19), and the cut between the curves $\kappa_{\min }^{\text {lepton }}(\lambda)$ and $\kappa_{-}^{\mathrm{NPB}}(\lambda)$ is sole and tangent. However, when the neutrino energy increases a bit (top right panel), the two different solutions $\lambda_{ \pm-}$given by Eq. (A24) appear, first as cuts between the curves $\kappa_{\min }^{\text {lepton }}(\lambda)$ and $\kappa_{-}^{\mathrm{NPB}}(\lambda)$. For higher neutrino energies, as that shown in the bottom left panel, the $\lambda_{+-}$cut given by Eq. (A24) occurs exactly at $\lambda=\lambda_{\max }$, and this happens for the reduced neutrino energy $\tilde{\epsilon}_{\nu}$ given in Eq. (20). Finally, in the bottom right panel we show the situation for a bit larger neutrino energy. In this case, the $\lambda_{+-}$solution given by Eq. (A24) corresponds to a cut point between the curves $\kappa_{-}^{\mathrm{NPB}}(\lambda)$ and $\kappa_{\max }^{\text {lepton }}(\lambda)$. 
well. However, there is a reduced neutrino energy $\epsilon_{\nu}$ for which $\lambda_{+-}=\lambda_{--}$. This happens when the radicand of Eq. (A24) is zero. Thus, we can find the $\epsilon_{\nu}$ value for this to happen by equating the radicand of Eq. (A24) to zero and solving the second degree equation for $\epsilon_{\nu}$. The result is

$$
\tilde{\epsilon}_{\nu \pm}=\frac{\tilde{m}_{\mu}\left(\tilde{m}_{\mu} \pm 1\right)}{\epsilon_{F}^{\prime}-\eta_{F}^{\prime}}
$$

where $\epsilon_{F}^{\prime} \equiv 1+\left(\epsilon_{F}-1\right) \psi_{\text {right }}^{2}$ and $\eta_{F}^{\prime}=\sqrt{\epsilon_{F}^{\prime 2}-1}$. Of course, if one wants to recover the results of the RFG, one substitutes $\psi_{\text {right }}=1$.

In principle, the $\tilde{\epsilon}_{\nu-}$ solution can be ruled out for electron and muon neutrinos because it is negative [94], but $\tilde{\epsilon}_{\nu+}$ is positive and must be considered. At this reduced neutrino energy $\tilde{\epsilon}_{\nu+}$, there is a single and tangent cut between the curves $\kappa_{\min }^{\text {lepton }}(\lambda)$ and $\kappa_{-}^{\mathrm{NPB}}(\lambda)$, as it can be seen in the top left panel of Fig. 15.

If the neutrino energy continues increasing, the two $\lambda$-cuts given by Eq. (A24) are different, but still both cuts occur between the $\kappa_{\min }^{\text {lepton }}(\lambda)$ and $\kappa_{-}^{\mathrm{NPB}}(\lambda)$ curves (as it can be observed in the top right panel of Fig. 15), until a higher neutrino energy $\left(\tilde{\epsilon}_{\nu}\right)$ is reached, at which $\lambda_{+-}=\lambda_{\max }$, with $\lambda_{\max }$ given by Eq. (12). In this range of values for $\epsilon_{\nu} \in\left[\tilde{\epsilon}_{\nu+}, \tilde{\epsilon}_{\nu}\right]$, the range of integration in $\lambda$ still runs from $\lambda \in\left[0, \lambda_{\max }\right]$.

To find $\tilde{\epsilon}_{\nu}$ one could equate $\lambda_{+-}=\lambda_{\max }$ and try to solve it for $\epsilon_{\nu}$, but this is very difficult because the equation turns out to be a third degree equation in $\epsilon_{\nu}$. Nonetheless, there is a very easy way to obtain this value of $\tilde{\epsilon}_{\nu}$ : we can equate $\kappa_{\max }^{\text {lepton }}\left(\lambda_{\max }\right)=\kappa_{-}^{\mathrm{NPB}}\left(\lambda_{\max }\right)$ and solve it for $\epsilon_{\nu}$. Given that $\kappa_{\max }^{\text {lepton }}\left(\lambda_{\max }\right)=\epsilon_{\nu}$, we can take Eq. (9) for $\kappa_{-}^{\mathrm{NPB}}\left(\lambda_{\max }\right)$ and solve the equation for $\epsilon_{\nu}$. The result is straightforward:

$$
\tilde{\epsilon}_{\nu}=\frac{\tilde{m}_{\mu}\left(\epsilon_{F}^{\prime}-\tilde{m}_{\mu}\right)}{\epsilon_{F}^{\prime}-\eta_{F}^{\prime}-2 \tilde{m}_{\mu}} .
$$

This is the situation shown in the bottom left panel of Fig. 15. And now, we can ensure that for $\epsilon_{\nu}>\tilde{\epsilon}_{\nu}$, the $\lambda_{+-}$ cut given by Eq. (A24) is lesser than $\lambda_{\max }$, but now it is a cut between the curve $\kappa_{-}^{\mathrm{NPB}}(\lambda)$ and the upper branch of the lepton kinematics boundary, $\kappa_{\max }^{\text {lepton }}(\lambda)$. This can be observed in the bottom right panel of Fig. 15.

Now, the integration range in the $\lambda$ variable is further constrained to be $\lambda \in\left[0, \lambda_{+-}\right]$where $\lambda_{+-}<\lambda_{\max }$ (only valid when $\epsilon_{\nu}>\tilde{\epsilon}_{\nu}$ ). Thus we can integrate the double differential cross section $\frac{d^{2} \sigma}{d \kappa d \lambda}$ in the region of the phase space where it is truly different from zero, thus making the integration algorithm the most efficient as possible. The integrated total CCQE cross section can now be written

$$
\sigma\left(E_{\nu}\right)=\int_{0}^{\lambda_{u}} d \lambda \int_{\kappa_{d}(\lambda)}^{\kappa_{u}(\lambda)} d \kappa \frac{d^{2} \sigma}{d \kappa d \lambda}\left(E_{\nu}\right)
$$

where

$$
\begin{aligned}
\lambda_{u} & = \begin{cases}\lambda_{\max } & \text { if } \epsilon_{\nu} \leq \tilde{\epsilon}_{\nu}, \\
\lambda_{+-} & \text {if } \epsilon_{\nu}>\tilde{\epsilon}_{\nu} ;\end{cases} \\
\kappa_{d}(\lambda) & =\max \left(\kappa_{-}^{\mathrm{NPB}}(\lambda), \kappa_{\min }^{\text {lepton }}(\lambda)\right), \\
\kappa_{u}(\lambda) & =\min \left(\kappa_{+}^{\mathrm{NPB}}(\lambda), \kappa_{\max }^{\text {lepton }}(\lambda)\right) .
\end{aligned}
$$

In Fig. 16 we show the results for the total CCQE integrated cross section off ${ }^{12} \mathrm{C}$ for the two models discussed in this work: RFG (solid line) and SuSAM* (short-dashed line). The left panel is for muon neutrino scattering, while the right one corresponds to muon antineutrino. We have displayed the uncertainty band of the $\mathrm{SuSAM}^{*}$ model, taken as the area between the predictions for the total cross sections obtained by taking the $f_{\mathrm{SuSAM} *}^{\max m}(\psi)$ scaling functions depicted in Fig. 7, instead of taking the $f_{\mathrm{SuSAM} *}^{\mathrm{central}}(\psi)$ scaling function of the same figure, which is the one we have used throughout this article. To compare with another important and relevant scaling model, already incorporated in GENIE [95], the SuSAv2-MEC model of Ref. [96], we have plotted the curve of this model in Fig. 16 in dot-dashed style as well.

In Fig. 16 we also display, in the form of a very narrow band of points surrounding the SuSAM* central curve, a Monte Carlo band that has been obtained by choosing an uniformly distributed value for the scaling function between the minimum and the maximum scaling functions of the SuSAM* model (Fig. 7) for each kinematic point $\psi(\lambda, \kappa)$ in the integration procedure for each neutrino/ antineutrino energy. Although it is almost imperceptible in the scale of Fig. 16, for each neutrino/antineutrino energy, there are 12 dots forming this extremely narrow band. Only for the higher and higher energies the spread of these points starts to become appreciable. This can be seen in Fig. 17, where a zoom view of this band is shown, exhibiting that the scale of fluctuations of this Monte Carlo band is much thinner than that shown in Fig. 16, where it is seen almost as a single curve without spreading.

An argument in favor of choosing an uniform distribution to draw a value for the scaling function for each kinematic point being integrated is that, in the superscaling analysis performed in Ref. [52], the superscaling band was found almost equally populated between the minimum and maximum scaling functions of the SuSAM* model. In fact, that band was obtained by using a density criterion, i.e., by keeping those points with at least more than a fixed number of neighboring points inside a circle of given radius.

The main conclusion that can be drawn by comparing the curves of the three different scaling models (RFG, $\mathrm{SuSAM}^{*}$, and SuSAv2-MEC) is that all of them lie inside the uncertainty band of the SuSAM* model. It is true that this band is very large, but not so large if compared with the experimental uncertainties, which is even truer for the antineutrino total cross section (right panel of Fig. 16), 

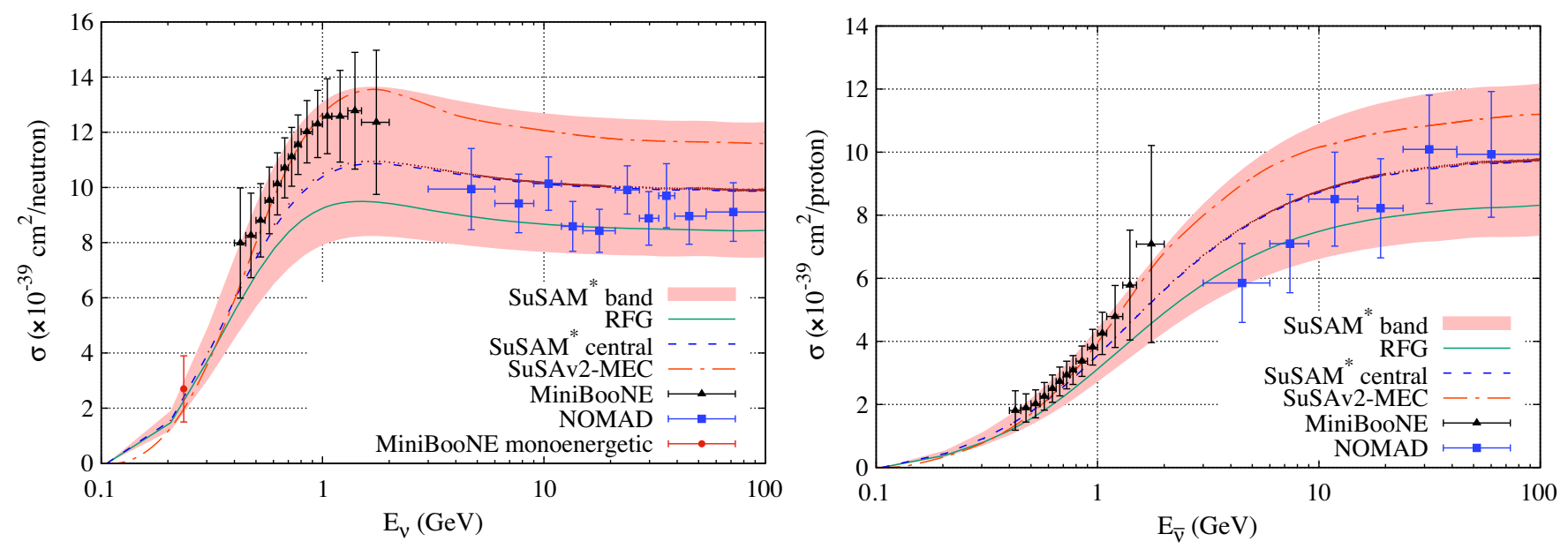

FIG. 16. Plot of the total CCQE cross section $\sigma\left(E_{\nu}\right)$ (normalized per interacting nucleon) as a function of the neutrino/antineutrino energy $E_{\nu(\bar{\nu})}$ for the two models discussed in this work: RFG (solid line) and SuSAM* (short-dashed line). In the left panel the neutrino total cross section per neutron off ${ }^{12} \mathrm{C}$ is displayed along with the experimental measurements of MiniBooNE [20,22] and NOMAD [97]. In the right panel, we show the same for the antineutrino total cross section per proton, compared with the measurements of MiniBooNE [21] and NOMAD [97] collaborations. For both models, RFG and SuSAM ${ }^{*}$, the nucleon relativistic effective mass $m_{N}^{*}=0.83 m_{N}$ has been taken, as well as a Fermi momentum of $k_{F}=212 \mathrm{MeV} / \mathrm{c}$, accordingly to the global fit to "QE" electron scattering data performed in Ref. [52]. Additionally, in dot-dashed style, it is also shown the SuSAv2-MEC model prediction, which has been taken from Ref. [96]. The Monte Carlo band (shown as points surrounding the SuSAM* central line) is also displayed (see main text for an explanation about how it has been obtained).

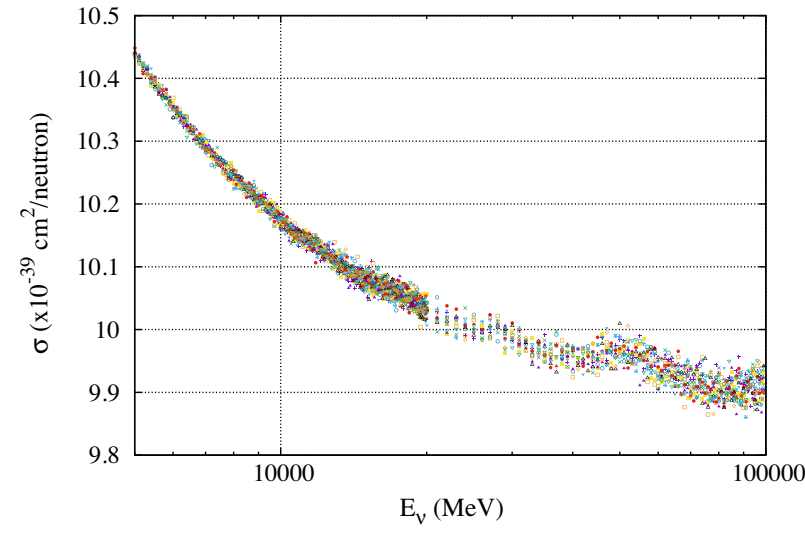

FIG. 17. Plot of the Monte Carlo band obtained as explained in the main text. Notice that the spreading of this band occurs in a scale much thinner than that displayed in Fig. 16.

where one can see that the theoretical uncertainty of the $\mathrm{SuSAM}^{*}$ model is of the same order as the error bars of the experimental points. In fact, the central prediction of the SuSAM* model is in between those of the RFG and the SuSAv2-MEC, and as observed in Fig. 16, it passes closer to both sets of the experimental data shown in the figure. These are the results of MiniBooNE [20-22] (for intermediate neutrino energies and for the new technique based on kaon decay at rest [20]) and NOMAD [97] (for the high neutrino energy range) experiments. It is worth another remark: the $\mathrm{SuSAM}^{*}$ predictions (band and central curve) are solely based on the superscaling properties of the selected "QE" electron scattering data out of the total inclusive $\left(e, e^{\prime}\right)$ data, in the global fit carried out in Ref. [52], and no CCQE neutrino scattering parameter has been fitted at all. The same can be said for the SuSAv2-MEC model, which is based on another scaling function [98] and with the contribution of the weak charged meson-exchange currents (MEC) calculated in Ref. [99]. The SuSAv2-MEC model describes the MiniBooNE data very well, but systematically overestimates the cross section at the NOMAD energies. In fact, this could point to a conflict between the MEC contribution used in this model and the NOMAD data.

It is not the purpose of this work to discuss the discrepancies between both sets of data shown in Fig. 16, because the experimental collaborations recognize in their works [21] that the experiments use different detector technologies and assume different topologies in defining CCQE events. And, in addition, the neutrino/ antineutrino energy drawn in the abscissa axes of Fig. 16 is the true neutrino energy, while in the experiments the energy is the reconstructed one (except for the kaon decay at rest technique), which assumes an educated guess to obtain it from the measured final lepton kinematic variables via an unfolding procedure. In fact, the problems related to the reconstruction of the neutrino energy have been addressed in a series of articles [100-111].

The total integrated CCQE cross sections shown in Fig. 16 have been obtained with the set of parameters $\left(k_{F}=212 \mathrm{MeV} / \mathrm{c}\right.$ and $\left.M^{*}=m_{N}^{*} / m_{N}=0.83\right)$ for ${ }^{12} \mathrm{C}$ obtained in the global fit to "QE" electron scattering data of Ref. [52], and given in Table II of the same reference. 
We did not try to adjust these and any other parameters of the model (e.g., axial mass of the nucleon) to the neutrino data. This is important to be stressed, because in the previous figures and formulas of this work, we have utterly used the values of $k_{F}=225 \mathrm{MeV} / \mathrm{c}$ and $M^{*}=1$ for the Fermi momentum and the relativistic effective mass, respectively. We used these values in the calculations of the previous figures because we did not want to bother the reader with additional complications related to the underlying Walecka model $[66,67]$ (see also Refs. [68,69]) in which the SuSAM* approach is based. It is also worth warning the reader that in this work we have used the usual dipole axial-vector form factor with a charged-current axial-vector mass of $M_{A}=1.008 \mathrm{GeV}$, and the set of vector form factors taken from the Galster parametrization given in Ref. [112]. This value of $M_{A}$ was obtained in a global fit to all available self-consistent data on CCQE (anti)neutrino scattering on nuclei, within the Smith-Monitz RFG model [44] and so-called running axial-vector mass of nucleon [113]. Since $M_{A}$ is an effective model-dependent parameter, we plan to adjust it from a global fit within the SuSAM* model.

It is worth noting that all the formulas appearing in this work can be translated to the real SuSAM* model by just changing the value of the Fermi momentum and the free nucleon mass $m_{N} \rightarrow m_{N}^{*}$, where $m_{N}^{*}$ is the value of the relativistic effective mass. These changes affect the values of $\eta_{F}, \epsilon_{F}, \epsilon_{F}^{\prime}, \eta_{F}^{\prime} \ldots$, but the form of the equations obtained in this work remains the same. Of course, what also does not change at all is the form of the scaling functions shown in Fig. 7. What changes is the value of the scaling variable $\psi$ for a given kinematics $(\omega, q)$, but not the form of the scaling functions.

What can also be stressed from the inspection of Fig. 16 is the effect of nuclear correlations in the integrated cross

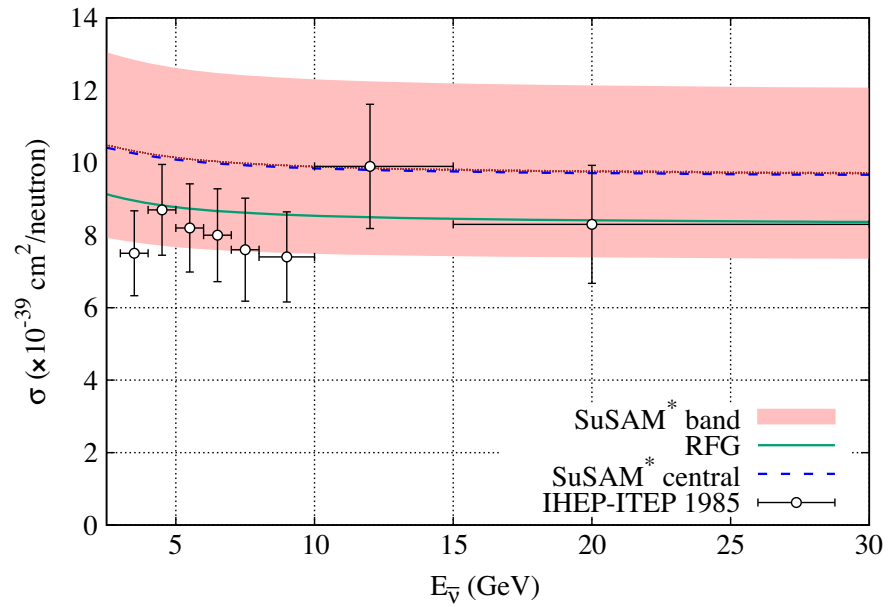

section. The RFG model does not contain nuclear correlations, not either high momentum components in its nuclear ground state. However, the SuSAM* model does contain them phenomenologically, because its scaling function has been fitted to a selected sample of "QE" electron scattering data extracted from the inclusive $\left(e, e^{\prime}\right)$ reaction data from a large list of different nuclear targets (see Ref. [52]). Therefore, the SuSAM* contains high momentum components in its nuclear model, although phenomenologically. In fact, the tails of the SuSAM* scaling function, that extend beyond $\psi= \pm 1$, partially account for these high momentum components, producing the enlarging of the available phase space if compared with the RFG (see in particular the right panel of Fig. 8, where the cut of the upper boundary of the SuSAM* model with the $\kappa$-axis occurs at $\kappa=\eta_{F}^{\prime}$, which can be considered as playing the role of an effective higher Fermi momentum). One can also compare the left panels of Figs. 10 and 11, or the left and right panels of Fig. 14 for CCQE antineutrino scattering, where one can observe that for the SuSAM* model, beyond the boundaries of the RFG denoted by the dot-dashed lines, there is still a significant region of the phase space with a non-negligible contribution to the double differential cross section. This is mainly responsible of the enhancement observed in the total cross section in both panels of Fig. 16 for the SuSAM* model with respect to the RFG.

The bands in Fig. 16 have been obtained by integrating the differential cross sections calculated with the minimum and maximum scaling functions fitted to the $\mathrm{QE}$ electron data and shown in Fig. 7. But the width of these bands do not necessarily correspond to the theoretical error, but an upper bound of it. The determination of the theoretical error in the total cross section is not the objective of this work. Nonetheless, we have examined a possible way to estimate

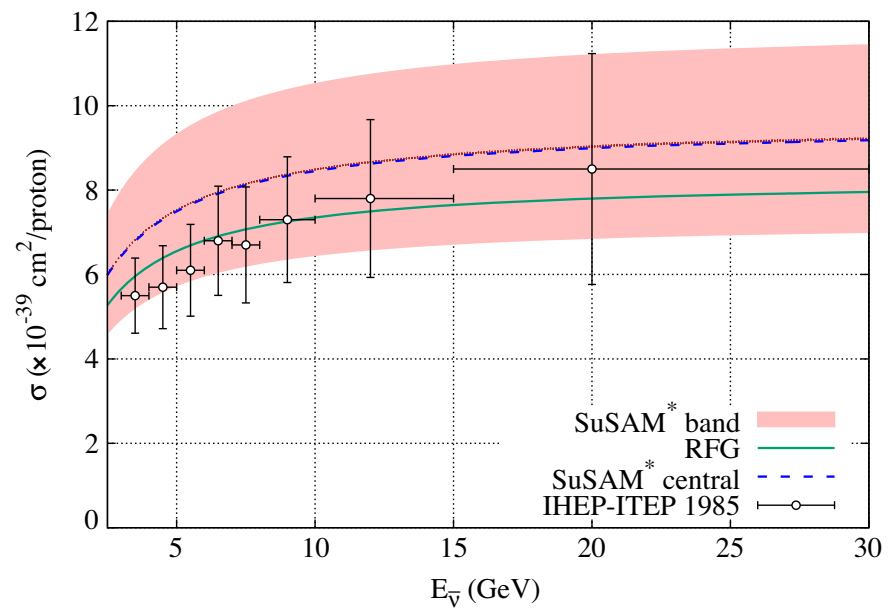

FIG. 18. Same as Fig. 16 but for muon (anti)neutrino, (right) left panel, CCQE scattering off ${ }^{27}$ Al. The experimental points are taken from the experiment of Ref. [12]. The values taken for the theoretical calculations of the RFG and SuSAM* models are $k_{F}=233 \mathrm{MeV} / \mathrm{c}$ for the Fermi momentum and $m_{N}^{*}=0.80 m_{N}$ for the relativistic effective mass (see "Global fit" parameters in Table II of Ref. [52]). 

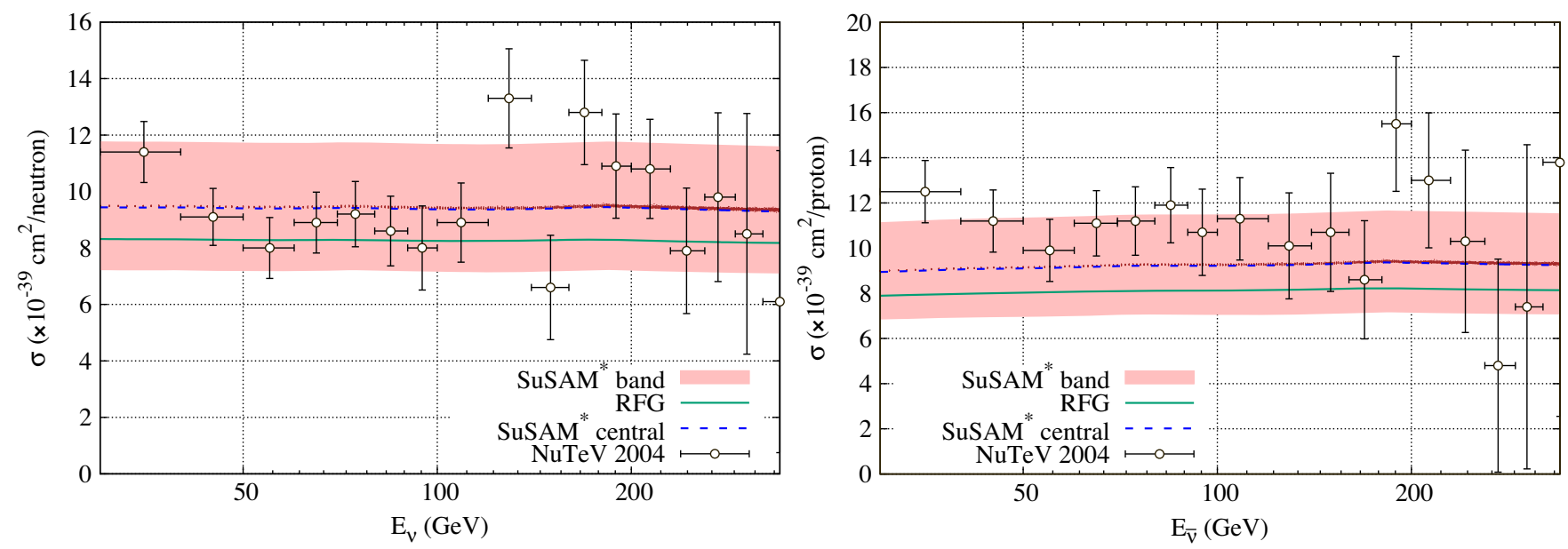

FIG. 19. Same as Figs. 16 and 18 but for muon (anti)neutrino, (right) left panel, CCQE scattering off ${ }^{56} \mathrm{Fe}$. The experimental points are taken from Ref. [114]. The values taken for the theoretical calculations of the RFG and SuSAM* models are $k_{F}=240 \mathrm{MeV} / \mathrm{c}$ for the Fermi momentum and $m_{N}^{*}=0.72 m_{N}$ for the relativistic effective mass (see "Global fit" parameters in Table II of Ref. [52]).

this error statistically, shown in Fig. 17, corresponding to the cross section of neutrinos between 5000 and $100000 \mathrm{MeV}$.

For a fixed energy of the neutrino we perform the numerical integration over $q$ and $\omega$ using random values for the scaling function $f(\psi)$ within the band. That is, we choose $f(\psi)=x$, where $x$ is a random value between $f_{\min }(\psi)$ and $f_{\max }(\psi)$ with an uniform probability distribution. A value of $x$ is sampled for each kinematics $(q, \omega)$ inside the integral. In this way, we estimate the statistical error as if we randomly chose experimental points within the quasielastic band. Repeating the calculation of the cross section many times, we obtain a series of points that form the band shown in Fig. 17. We see that now the dispersion of the points, which is the statistical error calculated, is much smaller than the width of the bands of Fig. 16.

Finally, in Figs. 18 and 19 we show similar results as those of Fig. 16 for older experiments [12,114] using ${ }^{27} \mathrm{Al}$ and ${ }^{56} \mathrm{Fe}$ targets, respectively. The comparison of the SuSAM* $^{*}$ band with the experimental results of Ref. [12], shown in Fig. 18 for muon neutrinos (left panel) and muon antineutrinos (right panel), seems to indicate that these data are closer to the lower bounds of the band, accumulating along the RFG curve. Nonetheless, the error bars are large enough, especially for the antineutrino induced CCQE reactions, to conclude the same than in Fig. 16, i.e., that the size of the vertical error bars is similar to the theoretical uncertainty derived from the SuSAM* model for the total CCQE cross sections as a function of the neutrino/ antineutrino energy, $\sigma\left(E_{\nu(\bar{\nu})}\right)$. Similar conclusions can be drawn by inspecting Fig. 19, except that for CCQE muon antineutrino scattering (right panel), the experimental measurements have a trend to accumulate along the upper bounds of the SuSAM* band, but with very large uncertainties.

\section{CONCLUSIONS}

In this work we have thoroughly analyzed the analytical boundaries of the phase space for the CCQE double differential cross section $\frac{d^{2} \sigma}{d \kappa d \lambda}$ within the scaling formalism for the RFG model, where these boundaries can be more easily obtained. This allows to perform the integration of this double differential cross section only in the region where it is truly different from zero, thus making the integration algorithm as efficient as possible. We have also easily extended the formalism to accommodate the SuSAM* model as well, taking into account the tails of the scaling function.

We have analyzed these double differential cross sections for CCQE muon neutrino and antineutrino scattering off ${ }^{12} \mathrm{C}$ at several neutrino/antineutrino energies as a benchmark. Our results show that the $\frac{d^{2} \sigma}{d \kappa d \lambda}$ cross section has very good properties to be implemented in the MC neutrino event generators, basically because of two main reasons: it is quite flat regardless of the neutrino energy; and it has a significant contribution in a larger region of the available phase space if compared with the usual $\frac{d^{2} \sigma}{d T_{\mu} d \cos \theta_{\mu}}$ cross section, especially at very high neutrino energies $E_{\nu(\bar{\nu})} \gtrsim 10-20 \mathrm{GeV}$. We think these features of the $\frac{d^{2} \sigma}{d \kappa d \lambda}$ cross section make it especially well suited to be used to generate final lepton events in any MC generator that uses the acceptance-rejection method.

We have used the analytical boundaries obtained in this work to integrate the double differential cross section in order to obtain the CCQE total integrated $\sigma\left(E_{\nu(\bar{\nu})}\right)$ cross section for the two models studied in this work: RFG and SuSAM*. The effect of the tails of the phenomenological SuSAM* scaling function, that partially account for nuclear correlations in the model, are directly responsible of an 
enhancement of about a $17-18 \%$ for intermediate and high neutrino energies. The same conclusion can be drawn for CCQE antineutrino scattering with roughly the same enhancement in percentage.

Finally, we have also compared the fully integrated CCQE total cross section in the RFG and SuSAM* models with past measurements carried out by several experiments using targets of ${ }^{12} \mathrm{C},{ }^{27} \mathrm{Al}$ and ${ }^{56} \mathrm{Fe}$. The main conclusion here is that all these measurements lie inside the uncertainty band of the SuSAM* model, being these uncertainties of roughly the same size as the experimental error bars. Nonetheless, we interpret the large uncertainty band of the SuSAM* model as lower and upper bounds for the true theoretical error in the total CCQE neutrino/antineutrino cross sections, based solely on our scaling analysis of electron scattering data [52]. The reader, by no means, should have the impression that this uncertainty band reflects the true error, just that the true error must be inside the band. This last statement, at first sight, can seem futile; but the experimental errors are also large, and even with that, different sets of data can become incompatible with the others.

Future works can be done based on the findings of this study. In particular, we are working on the study of how nuclear correlations can be approximately and phenomenologically incorporated in the RFG model.

\section{ACKNOWLEDGMENTS}

This work has been partially supported by the former Spanish Ministerio de Economia y Competitividad and ERDF (European Regional Development Fund) under Contract No. FIS2017-85053-C2-1P, by the Junta de Andalucía Grant No. FQM225, by Contract No. PID2020114767 GB-I00 funded by MCIN/ AEI /10.13039/ 501100011033, and by the Russian Science Foundation Grant No. 18-12-00271.

\section{APPENDIX A: ANALYTICAL FORMULAS FOR THE BOUNDARIES IN THE RFG MODEL}

The RFG model requires that $\epsilon_{0} \leq \epsilon_{F}$, otherwise the scaling variable would take values $|\psi|>1$. The physical constraint of $\tau \geq 0$ implies that we have to search only in the region where $\kappa \geq \lambda$. Finally, it can be shown that if $\kappa \geq \eta_{F}$ (or $q \geq 2 k_{F}$, which corresponds to the NPB region) then $\epsilon_{0}=\kappa \sqrt{1+1 / \tau}-\lambda \geq \epsilon_{F}-2 \lambda$ for all positive values of the energy transfer $\lambda[115,116]$. In the NPB region, typically $q \gtrsim 500 \mathrm{MeV} / \mathrm{c}$, and the above condition can be rewritten as $\kappa \gtrsim 1 / 4$.

As discussed in Sec. III A, the boundaries of the RFG scaling variable $(-1,+1)$ are reached when $\epsilon_{0}=\epsilon_{F}$ as it follows from Eq. (6). Solving the equation $\epsilon_{0}=$ $\kappa \sqrt{1+1 / \tau}-\lambda=\epsilon_{F}$ in the NPB region (corresponding to $\left.\kappa \geq \eta_{F}\right)$ we get two different curves in the $(\lambda, \kappa)$ plane. One of them, $\kappa_{+}^{\mathrm{NPB}}(\lambda)$, is always greater than $\kappa_{\mathrm{QE}}(\lambda)$ and corresponds to $\psi=-1$, just because $\kappa_{+}^{\mathrm{NPB}}(\lambda)>$ $\sqrt{\lambda(\lambda+1)}$ and this implies $\lambda<\tau$ :

$$
\begin{aligned}
\kappa_{+}^{\mathrm{NPB}}(\lambda)>\kappa_{\mathrm{QE}}(\lambda) & \equiv \sqrt{\lambda(\lambda+1)} \geq 0 \\
& \Leftrightarrow\left(\kappa_{+}^{\mathrm{NPB}}(\lambda)\right)^{2}-\lambda^{2}>\lambda \\
& \Leftrightarrow \tau_{+}^{\mathrm{NPB}}(\lambda)>\lambda \\
& \Rightarrow \operatorname{sign}\left(\lambda-\tau_{+}^{\mathrm{NPB}}(\lambda)\right)=-1 .
\end{aligned}
$$

Therefore, along the curve $\kappa_{+}^{\mathrm{NPB}}(\lambda)$ the scaling variable is always equal to -1 . Analogously, there is another curve, a solution of $\epsilon_{0}=\epsilon_{F}$, called $\kappa_{-}^{\mathrm{NPB}}(\lambda)$, which is always less than $\kappa_{\mathrm{QE}}(\lambda)$, and where [by similar arguments as those proven in Eq. (A1)] the scaling variable is always equal to +1 .

The expressions of these two curves, $\kappa_{ \pm}^{\mathrm{NPB}}(\lambda)$, are given below in two different ways in the Appendices A 1 and A 2.

\section{Obtaining $\kappa_{ \pm}^{\mathrm{NPB}}(\lambda)$}

One of the easiest ways to obtain the limiting curves $\kappa_{ \pm}^{\mathrm{NPB}}(\lambda)$ can be found in Eq. (A.2) of Appendix A of Ref. [117] (see also Eqs. (C7)-(C9) of Ref. [8]). This latter equation allows to find the lowest and highest $\omega$ limits for fixed $q$. These two limits can be found from the equation $\epsilon_{0}=\epsilon_{F}$ :

$$
\omega_{ \pm}=E_{k_{F} \pm q}-E_{F}=\sqrt{\left(k_{F} \pm q\right)^{2}+m_{N}^{2}}-\sqrt{k_{F}^{2}+m_{N}^{2}} .
$$

Dividing on both sides of the above equations by $2 m_{N}$, and writing everything in terms of the dimensionless variables given in Eqs. (4) and (5), we obtain the boundaries in $\lambda_{ \pm}$for fixed $\kappa$ :

$$
\lambda_{ \pm}(\kappa)=\frac{1}{2} \sqrt{\left(\eta_{F} \pm 2 \kappa\right)^{2}+1}-\frac{1}{2} \epsilon_{F} .
$$

The problem with the boundaries given in (A2) is that they are given as curves $\lambda=\lambda(\kappa)$, whereas we want them in the form of curves $\kappa=\kappa(\lambda)$. Thus, we have to find the inverse functions. Then, writing $\lambda_{+}(\kappa) \equiv$ $\frac{1}{2} \sqrt{\left(\eta_{F}+2 \kappa\right)^{2}+1}-\frac{1}{2} \epsilon_{F}=\lambda$, and solving for $\kappa$, we obtain the lower bound $\kappa_{-}^{\mathrm{NPB}}(\lambda)$, which is given by

$$
\kappa_{-}^{\mathrm{NPB}}(\lambda)=\frac{1}{2} \sqrt{\left(\epsilon_{F}+2 \lambda\right)^{2}-1}-\frac{\eta_{F}}{2} .
$$

In Fig. 20 one can inspect that the inversion of $\lambda_{-}(\kappa) \equiv$ $\frac{1}{2} \sqrt{\left(\eta_{F}-2 \kappa\right)^{2}+1}-\frac{1}{2} \epsilon_{F}=\lambda$ needs a bit of care because it must be solved for $\kappa$ in the region where $\lambda \geq 0$.

$$
\begin{aligned}
\lambda_{-}(\kappa)=\lambda & \Leftrightarrow\left(\eta_{F}-2 \kappa\right)^{2}=\left(2 \lambda+\epsilon_{F}\right)^{2}-1 \\
& \Leftrightarrow\left|\eta_{F}-2 \kappa\right|=\sqrt{\left(\epsilon_{F}+2 \lambda\right)^{2}-1} ;
\end{aligned}
$$




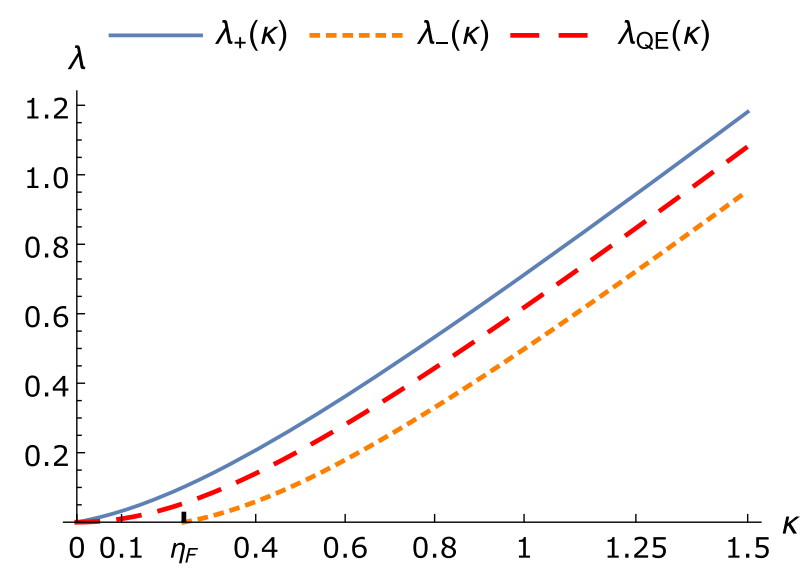

FIG. 20. Plot of the two limiting curves $\lambda_{ \pm}(\kappa)$ as a function of $\kappa$ in the RFG model in the NPB region, i.e., for $\kappa \geq \eta_{F}$ [notice that for $\kappa<\eta_{F}$ the $\lambda_{-}(\kappa)$ curve reaches negative values, which are forbidden; this is because it is entering into the PB region]. In this figure, we have taken $\eta_{F}=0.239$. The dashed curve $\lambda_{\mathrm{QE}}(\kappa)=$ $-\frac{1}{2}+\frac{1}{2} \sqrt{1+4 \kappa^{2}}$ corresponds to the inverse of $\kappa_{\mathrm{QE}}(\lambda)=\kappa$.

if $\kappa \geq \eta_{F}$ then $\left|\eta_{F}-2 \kappa\right|=2 \kappa-\eta_{F}$ and thus Eq. (A4) becomes

$$
\kappa_{+}^{\mathrm{NPB}}(\lambda)=\frac{1}{2} \sqrt{\left(\epsilon_{F}+2 \lambda\right)^{2}-1}+\frac{\eta_{F}}{2} .
$$

Eqs. (A3) and (A5) are plotted as the thick solid and shortdashed lines of Fig. 1.

\section{Alternative form of obtaining $\boldsymbol{\kappa}_{ \pm}^{\mathrm{NPB}}(\lambda)$}

In the NPB region, it is true that $\epsilon_{0}=\kappa \sqrt{1+1 / \tau}-\lambda$. The maximum value is $\epsilon_{0}=\epsilon_{F}$ and this last equation defines two curves in the $(\lambda, \kappa)$ plane. Taking the square and using $\tau=\kappa^{2}-\lambda^{2}$, we obtain the following biquadratic equation in $\kappa$ :

$$
\kappa^{4}-\left(2 \lambda^{2}+\phi\right) \kappa^{2}+\lambda^{2}\left(\lambda^{2}+\phi+1\right)=0,
$$

where $\phi=\epsilon_{F}\left(\epsilon_{F}+2 \lambda\right)-1$. Now, making a change in a variable $t \equiv \kappa^{2}$ we arrive to a quadratic equation

$$
t^{2}-\left(2 \lambda^{2}+\phi\right) t+\lambda^{2}\left(\lambda^{2}+\phi+1\right)=0,
$$

whose two roots are

$$
t_{ \pm}(\lambda)=\frac{2 \lambda^{2}+\phi \pm \sqrt{\phi^{2}-4 \lambda^{2}}}{2} .
$$

It can be easily shown that the discriminant of Eq. (A7) is always positive for $\lambda \geq 0$, because it can be written as

$\phi^{2}-4 \lambda^{2}=\eta_{F}^{2}\left(4 \lambda^{2}+4 \lambda \epsilon_{F}+\eta_{F}^{2}\right)>0 \quad$ if $\lambda \geq 0$.
This ensures that the roots $t_{ \pm}(\lambda)$ are real. With this, we can write Eq. (A6) as

$$
\left[\kappa^{2}-t_{+}(\lambda)\right]\left[\kappa^{2}-t_{-}(\lambda)\right]=0 .
$$

It is also easy to demonstrate that both roots $t_{ \pm}(\lambda)$, besides being real, are also positive. To this end we first write the negative of the coefficient of $t$ in Eq. (A6) as

$$
2 \lambda^{2}+\phi=2 \lambda^{2}+2 \lambda \epsilon_{F}+\eta_{F}^{2}>0 \quad \text { if } \lambda \geq 0 .
$$

With this, it is obvious that $t_{+}(\lambda)$ is positive for $\lambda \geq 0$. To demonstrate the same for $t_{-}(\lambda)$, it is enough to prove that the square of Eq. (A10) is greater than the discriminant given in Eq. (A8). This comes from

$$
\begin{aligned}
\left(2 \lambda^{2}+2 \lambda \epsilon_{F}+\eta_{F}^{2}\right)^{2} & \geq \eta_{F}^{2}\left(4 \lambda^{2}+4 \lambda \epsilon_{F}+\eta_{F}^{2}\right) \Leftrightarrow \\
4 \lambda^{2}\left(\lambda^{2}+2 \epsilon_{F} \lambda+\epsilon_{F}^{2}\right) & \geq 0,
\end{aligned}
$$

which is true if $\lambda \geq 0$. With these proofs we can be sure that the four roots of $\kappa$ in Eq. (A9) are all real as well. This means that the boundary in the $(\lambda, \kappa)$-plane where $-1 \leq \psi \leq 1$ is bounded by the curves

$$
\kappa_{ \pm}^{\mathrm{NPB}}(\lambda)=\sqrt{\frac{\left(2 \lambda^{2}+\phi\right) \pm \sqrt{\phi^{2}-4 \lambda^{2}}}{2}},
$$

in the NPB region, i.e., for $\kappa \geq \eta_{F}$.

It could seem that these two curves given by Eq. (A11) are totally different from those obtained in Appendix A 1 and given in Eqs. (A3) and (A5), but they are actually the same, and already plotted in Fig. 1. One way of proving this is by raising to the square the $\kappa_{ \pm}^{\mathrm{NPB}}(\lambda)$ functions obtained in Appendix A 1, given by Eqs. (A3) and (A5); then an easy but lengthy algebra manipulation can demonstrate that the square of Eq. (A5) is equal to $t_{+}(\lambda)$ and that the square of Eq. (A3) is also equal to $t_{-}(\lambda)$, both jointly given in Eq. (A7).

\section{Obtaining $\boldsymbol{\kappa}_{ \pm}^{\mathrm{PB}}(\lambda)$}

Up to now we have been discussing the boundaries in the $(\lambda, \kappa)$-plane of the NPB region, where the following identity holds true:

$\epsilon_{0} \equiv \max \left(\kappa \sqrt{1+\frac{1}{\tau}}-\lambda, \epsilon_{F}-2 \lambda\right)=\kappa \sqrt{1+\frac{1}{\tau}}-\lambda$.

As it was stated above, Eq. (A12) always holds when $\kappa \geq \eta_{F}$, but not necessarily when $\kappa<\eta_{F}$. We will see below that for $\lambda \leq \kappa<\eta_{F}$ (since $\tau \geq 0$ ), there are some regions in the $(\lambda, \kappa)$-plane where $\epsilon_{0}$ can be equal to the second argument of the maximum function appearing in Eq. (A12), while there are other regions where $\epsilon_{0}$ is still equal to the first argument of the maximum function. 
In order to delimit these boundaries note that

$$
\kappa \sqrt{1+\frac{1}{\tau}}-\lambda \leq \epsilon_{F}-2 \lambda \Leftrightarrow \kappa \sqrt{1+\frac{1}{\tau}} \leq \epsilon_{F}-\lambda .
$$

The latter inequality defines some region in the $(\lambda, \kappa)$ plane. As both sides of the inequality given in Eq. (A13) are positive (because we are looking for solutions where $\lambda<\eta_{F}<\epsilon_{F}$ ), taking the square, substituting $\tau=\kappa^{2}-\lambda^{2}$, and rearranging terms we finish with another biquadratic inequality for $\kappa$ :

$$
\kappa^{4}-\rho \kappa^{2}+\left(\lambda \epsilon_{F}-\lambda^{2}\right)^{2} \leq 0,
$$

where $\rho=2 \lambda^{2}-2 \lambda \epsilon_{F}+\eta_{F}^{2}$. Performing the usual trick of solving the inequality by making the change of variable $u \equiv \kappa^{2}$, we obtain that the equality holds for

$$
u_{ \pm}(\lambda)=\frac{\rho \pm \sqrt{\rho^{2}-4\left(\lambda \epsilon_{F}-\lambda^{2}\right)^{2}}}{2} .
$$

For Eq. (A15) to have real solutions, the discriminant must be positive at least in the region of $\lambda$-values where we are seeking a solution.

It is not difficult to write the condition for the discriminant to be positive as

$$
\begin{aligned}
\rho^{2}-4\left(\lambda \epsilon_{F}-\lambda^{2}\right)^{2} & \geq 0 \Leftrightarrow \\
{\left[\rho-2\left(\lambda \varepsilon_{F}-\lambda^{2}\right)\right]\left[\rho+2\left(\lambda \varepsilon_{F}-\lambda^{2}\right)\right] } & \geq 0 \Leftrightarrow \\
4 \lambda^{2}-4 \lambda \epsilon_{F}+\eta_{F}^{2} & \geq 0 .
\end{aligned}
$$

The last equality has two roots for $\lambda$. They are

$$
\lambda_{ \pm}=\frac{\epsilon_{F} \pm 1}{2}>0 .
$$

Hence, the last inequality of Eq. (A16) can be written as

$$
4\left(\lambda-\lambda_{+}\right)\left(\lambda-\lambda_{-}\right) \geq 0 .
$$

The only meaningful solution to Eq. (A18) is that $\lambda \leq \lambda_{-}<$ $\lambda_{+}$[118]. The next step is to see if $\lambda_{-}$is lesser than $\eta_{F}$ or not, because if so then the interval in $\lambda$ where to have real roots for $u_{ \pm}(\lambda)$ is further constrained compared to the interval defined by $0 \leq \lambda<\eta_{F}$. Clearly,

$$
\begin{aligned}
\lambda_{-} \equiv \frac{\epsilon_{F}-1}{2} & =\frac{\sqrt{1+\eta_{F}^{2}}-1}{2} \\
& =\frac{\eta_{F}^{2}}{2\left(\sqrt{1+\eta_{F}^{2}}+1\right)}<\frac{\eta_{F}^{2}}{4}<\eta_{F},
\end{aligned}
$$

where the last inequalities hold because $0<\eta_{F}<1$, which implies that $\eta_{F}^{2}<\eta_{F}$. So we can conclude that the discriminant of Eq. (A15) is positive and $u_{ \pm}(\lambda)$ are real roots of Eq. (A14) for $0 \leq \lambda \leq \lambda_{-}<\eta_{F}$. The next step is wondering about the sign and magnitude of the coefficient of $\kappa^{2}$ in Eq. (A14) in the region of $\lambda$-values between 0 and $\lambda_{-}$. The reason for this is because depending upon its sign and magnitude, the roots $u_{ \pm}(\lambda)$ can be negative and we want them to be positive because $u_{ \pm}(\lambda)=\left[\kappa_{ \pm}^{\mathrm{PB}}(\lambda)\right]^{2}$ should be the square of real roots of Eq. (A14). To this end, we set out the following inequality and seek for their solutions:

$$
\rho \geq 0 .
$$

The equality $\rho=0$ has two positive roots for $\lambda$ :

$$
\lambda_{ \pm}^{\prime}=\frac{\epsilon_{F} \pm \sqrt{2-\epsilon_{F}^{2}}}{2}=\frac{\sqrt{1+\eta_{F}^{2}} \pm \sqrt{1-\eta_{F}^{2}}}{2}>0 .
$$

We have to compare them with $\lambda_{-}$, given in Eq. (A17). The reason for this is because if any of the two new roots $\lambda_{ \pm}^{\prime}$ is lesser than $\lambda_{-}$, then the interval in $\lambda$ where to seek the boundary of the PB region can be, again, further constrained from the last condition $0 \leq \lambda \leq \lambda_{-}$. It is easy to see that $\lambda_{+}^{\prime}$ is clearly greater than $\lambda_{-}$:

$$
\lambda_{+}^{\prime}=\frac{\epsilon_{F}+\sqrt{1-\eta_{F}^{2}}}{2}>\frac{\epsilon_{F}}{2}>\frac{\epsilon_{F}-1}{2} \equiv \lambda_{-} .
$$

On the other hand, it is also straightforward to see that $\lambda_{-}^{\prime}$ is greater than $\lambda_{-}$as well:

$$
\begin{aligned}
\lambda_{-}^{\prime} & \equiv \frac{\sqrt{1+\eta_{F}^{2}}-\sqrt{1-\eta_{F}^{2}}}{2} \\
& =\frac{\eta_{F}^{2}}{\sqrt{1+\eta_{F}^{2}}+\sqrt{1-\eta_{F}^{2}}}>\frac{\eta_{F}^{2}}{4}>\lambda_{-},
\end{aligned}
$$

where in the second step we have multiplied and divided by $\sqrt{1+\eta_{F}^{2}}+\sqrt{1-\eta_{F}^{2}}$.

Finally, it is worth noting that the inequality (A20) can be rewritten as

$$
2\left(\lambda-\lambda_{+}^{\prime}\right)\left(\lambda-\lambda_{-}^{\prime}\right) \geq 0,
$$

which is absolutely fulfilled if $\lambda \leq \lambda_{-}<\lambda_{-}^{\prime}<\lambda_{+}^{\prime}$, because then both parentheses in Eq. (A21) are negative and their product is positive. Having found the most restrictive region in the $\lambda$ variable where Eqs. (A16) and (A20) are simultaneously fulfilled, we can assert that the roots $u_{ \pm}(\lambda)$ given in Eq. (A15) are both real and positive [119]. Thus, we can rewrite Eq. (A14) as

$$
\left[\kappa^{2}-u_{+}(\lambda)\right]\left[\kappa^{2}-u_{-}(\lambda)\right] \leq 0 .
$$

From the above inequality, it is obvious that the only solution is 


$$
\sqrt{u_{-}(\lambda)} \leq \kappa \leq \sqrt{u_{+}(\lambda)}
$$

where we have taken the square roots because the solutions $u_{ \pm}(\lambda)$ and $\kappa$ are all positive. Therefore, we can conclude from all this discussion that the region where PB makes $\epsilon_{0}$ to be equal to the second argument, $\epsilon_{F}-2 \lambda$, of the maximum function displayed in Eq. (A12), corresponds to the region $\kappa_{-}^{\mathrm{PB}}(\lambda) \leq \kappa \leq \kappa_{+}^{\mathrm{PB}}(\lambda)$ in the region where $0 \leq \lambda \leq \lambda_{-}$with

$$
\kappa_{ \pm}^{\mathrm{PB}}(\lambda)=\sqrt{\frac{\rho \pm \sqrt{\rho^{2}-4\left(\lambda \epsilon_{F}-\lambda^{2}\right)^{2}}}{2}} .
$$

It is also easy to find the values of $\kappa_{ \pm}^{\mathrm{PB}}(\lambda)$ for $\lambda=0$ and $\lambda=\lambda_{-}$. They are

$$
\begin{gathered}
\kappa_{ \pm}^{\mathrm{PB}}\left(\lambda_{-}\right)=\frac{\eta_{F}}{2} \\
\kappa_{-}^{\mathrm{PB}}(0)=0, \quad \kappa_{+}^{\mathrm{PB}}(0)=\eta_{F} .
\end{gathered}
$$

The first two above equations can be easily found by noticing that, for $\lambda=\lambda_{-}=\left(\epsilon_{F}-1\right) / 2$, the discriminant of $u_{ \pm}(\lambda)$ is exactly zero [see Eq. (A18)], and then there is no difference between $\kappa_{+}^{\mathrm{PB}}\left(\lambda_{-}\right)$and $\kappa_{-}^{\mathrm{PB}}\left(\lambda_{-}\right)$. It is also easy to notice that $\kappa_{\mathrm{QE}}\left(\lambda_{-}\right)=\eta_{F} / 2$ :

$$
\kappa_{\mathrm{QE}}\left(\lambda_{-}\right)=\sqrt{\lambda_{-}\left(\lambda_{-}+1\right)}=\sqrt{\frac{\epsilon_{F}^{2}-1}{4}}=\frac{\eta_{F}}{2} .
$$

This means that for $\lambda=\lambda_{-}, \epsilon_{0}=\epsilon_{F}-2 \lambda_{-}=1$ and then the scaling variable at the point $(\lambda, \kappa)=\left(\lambda_{-}, \eta_{F} / 2\right)$ is exactly 0 [see definition given in Eq. (6)].

\section{Obtaining $\kappa_{\max , \min }^{\text {lepton }}(\lambda)$}

As both $\kappa_{ \pm}^{\mathrm{NPB}}(\lambda)$ curves are increasing functions of $\lambda$ (see for example Figs. 1, 3, or 4), it is interesting to look for the cutting points between $\kappa_{\max }^{\text {lepton }}(\lambda)$ and $\kappa_{ \pm}^{\mathrm{NPB}}(\lambda)$, or between $\kappa_{\min }^{\text {lepton }}(\lambda)$ and $\kappa_{ \pm}^{\mathrm{NPB}}(\lambda)$, if any. These cutting points will help us to constrain and to understand the form of the available phase space in the RFG model for a fixed neutrino/ antineutrino reduced energy $\epsilon_{\nu}$.

We can start by looking for the $\lambda$ value where $\kappa_{+}^{\mathrm{NPB}}(\lambda)=\kappa_{\max }^{\text {lepton }}(\lambda)$. This value can give us the $\lambda$ point where the minimum function appearing in expression (16) changes from selecting one curve to the other. In this case, to obtain the solution for $\lambda$, it is better to use the expression for $\kappa_{+}^{\mathrm{NPB}}(\lambda)$ given in Eq. (A5) rather than that given in Eq. (A11), although both are equivalent, just because the first one is much simpler to manipulate. To obtain the solution it is necessary to square twice the equation, and we finish with the following second degree equation for $\lambda$ after a lengthy algebra manipulation:

$$
a \lambda^{2}+b \lambda+c=0
$$

with

$$
\begin{aligned}
a & \equiv 1+4 \epsilon_{\nu}\left(\epsilon_{F}+\eta_{F}\right) \\
b & \equiv 2 \epsilon_{F}\left(\tilde{m}_{\mu}^{2}+\epsilon_{\nu} \eta_{F}\right)+2 \epsilon_{\nu}\left[\eta_{F}^{2}+2 \tilde{m}_{\mu}^{2}-2 \epsilon_{\nu}\left(\epsilon_{F}+\eta_{F}\right)\right], \\
c & \equiv \tilde{m}_{\mu}^{2}\left(\eta_{F}^{2}-2 \epsilon_{\nu} \eta_{F}+\tilde{m}_{\mu}^{2}\right) .
\end{aligned}
$$

The above equation has two roots. Only the solution with the positive square root is positive for some values of $\epsilon_{\nu}$. The other solution is always negative and we discard it. The relevant solution, which we call $\lambda_{++}$, is given by

$$
\begin{aligned}
\lambda_{++}= & \frac{\zeta_{+}\left(2 \epsilon_{\nu}-\eta_{F}\right)-\tilde{m}_{\mu}^{2}\left(\epsilon_{F}+2 \epsilon_{\nu}\right)}{\left(1+4 \zeta_{+}\right)} \\
& +\frac{\left|2 \epsilon_{\nu}-\eta_{F}\right| \sqrt{\tilde{m}_{\mu}^{4}-\tilde{m}_{\mu}^{2}+\zeta_{+}\left(\zeta_{+}-2 \tilde{m}_{\mu}^{2}\right)}}{\left(1+4 \zeta_{+}\right)},
\end{aligned}
$$

where we define $\zeta_{+}=\epsilon_{\nu}\left(\epsilon_{F}+\eta_{F}\right)$. In the above Eq. (A22) there is a value for the reduced neutrino energy $\epsilon_{\nu}$ for which $\lambda_{++}=0$. This value can be found by equating the numerator of (A22) to zero and solving for $\epsilon_{\nu}$. This value of $\epsilon_{\nu}$ is precisely that for which the cut point between $\kappa_{+}^{\mathrm{NPB}}(\lambda)$ and $\kappa_{\max }^{\text {lepton }}(\lambda)$ occurs at $\lambda=0$. Again, a lengthy and tedious algebraic manipulation leaves us with another second degree equation in the variable $\epsilon_{\nu}$ :

$$
a \epsilon_{\nu}^{2}+b \epsilon_{\nu}+c=0
$$

with

$$
\begin{aligned}
a & \equiv 8 \eta_{F}\left(\eta_{F}+\epsilon_{F}\right), \\
b & \equiv 2\left(\eta_{F}-2\left(\eta_{F}+\epsilon_{F}\right)\left(\eta_{F}^{2}+\tilde{m}_{\mu}^{2}\right)\right), \\
c & \equiv-\eta_{F}^{2}-\tilde{m}_{\mu}^{2} .
\end{aligned}
$$

Only the solution with the positive square root is again positive, while the other solution is always negative and we discard it. The meaningful solution is [120]

$$
\epsilon_{\nu_{+}}=\frac{\eta_{F}^{2}+\tilde{m}_{\mu}^{2}}{2 \eta_{F}}>0
$$

Notice that the solution given in the above Eq. (A23) depends both on the reduced final lepton mass and on a nuclear property, namely the Fermi momentum (in units of the nucleon mass).

Also note that, given the behavior of the curves $\kappa_{\max }^{\text {lepton }}(\lambda)$ (which is a monotonically decreasing function of $\lambda$, as already mentioned in Sec. III B), and $\kappa_{+}^{\mathrm{NPB}}(\lambda)$ (which is monotonically increasing), for $\tilde{m}_{\mu} \leq \epsilon_{\nu} \leq \epsilon_{\nu_{+}}$the upper limit of the phase space of the QE double differential cross 
section with respect to final lepton variables in the RFG model is bounded only by the curve $\kappa_{\max }^{\text {lepton }}(\lambda)$. Or said in other words, if $\tilde{m}_{\mu} \leq \epsilon_{\nu} \leq \epsilon_{\nu_{+}}$, then the minimum function of the right-hand side of inequality (16) is always the curve $\kappa_{\max }^{\text {lepton }}(\lambda)$ for all the allowed $\lambda$ values.

On the other hand, the presence of the reduced lepton mass in Eq. (A23) means that the necessary ranges of neutrino energies to allow the lepton kinematic constraints to determine by themselves the upper boundary of the phase space, depend a lot on the kind of neutrino flavor for charged current processes. For instance, for tau neutrinos and for typical values of Fermi momenta $\left(\eta_{F} \simeq 0.24\right)$, the neutrino energy $E_{\nu_{+}}=2 m_{N} \epsilon_{\nu_{+}} \simeq 3.72 \mathrm{GeV}$, which is already a quite large neutrino energy for the intermediate neutrino energy range. However, for muon neutrinos, $E_{\nu_{+}} \simeq 240 \mathrm{MeV}$, which is a quite low neutrino energy. Of course, these values are completely related to the threshold neutrino energies to produce a $\tau$ lepton or a muon in charged-current elastic scattering with nucleons, respectively.

Now we can look for the cut point between $\kappa_{\max }^{\text {lepton }}(\lambda)$ and $\kappa_{-}^{\mathrm{NPB}}(\lambda)$, which will occur for a $\lambda$ value larger [121] than $\lambda_{++}$, given in Eq. (A22). Again, after a lengthy calculation, solving $\kappa_{-}^{\mathrm{NPB}}(\lambda)=\kappa_{\max }^{\text {lepton }}(\lambda)$ for $\lambda$, we find two roots:

$$
\begin{aligned}
\lambda_{ \pm-}= & \frac{\zeta_{-}\left(2 \epsilon_{\nu}+\eta_{F}\right)-\tilde{m}_{\mu}^{2}\left(\epsilon_{F}+2 \epsilon_{\nu}\right)}{\left(1+4 \zeta_{-}\right)} \\
& \pm \frac{\left(2 \epsilon_{\nu}+\eta_{F}\right) \sqrt{\tilde{m}_{\mu}^{4}-\tilde{m}_{\mu}^{2}+\zeta_{-}\left(\zeta_{-}-2 \tilde{m}_{\mu}^{2}\right)}}{\left(1+4 \zeta_{-}\right)}
\end{aligned}
$$

where we define $\zeta_{-}=\epsilon_{\nu}\left(\epsilon_{F}-\eta_{F}\right)$. Both roots are physical (not complex numbers) for some reduced neutrino energies which depend on the model scaling function (see Fig. 15). Note that the expression for the first root, $\lambda_{+-}$, corresponds to the solution $\lambda_{++}$given in Eq. (A22) if one makes the replacement $\eta_{F} \mapsto-\eta_{F}$, which makes sense because the only difference between $\kappa_{+}^{\mathrm{NPB}}(\lambda)$ [given in Eq. (A5)], and $\kappa_{-}^{\mathrm{NPB}}(\lambda)$ [Eq. (A3)] is the sign of $\eta_{F}$. The other root, $\lambda_{--}$, when it is physical, always corresponds to the cutting point between the curves $\kappa_{-}^{\mathrm{NPB}}(\lambda)$ and $\kappa_{\min }^{\text {lepton }}(\lambda)$. This latter solution corresponds to the $\lambda$ point where the maximum function appearing on the left-hand side of the inequality (16) changes from one of its arguments to the other. It could seem striking at first glance that this solution appears when we have not used at all the $\kappa_{\min }^{\text {lepton }}(\lambda)$ curve to obtain it, but (as it can be seen from Fig. 6) the curves $\kappa_{\text {max,min }}^{\text {lepton }}(\lambda)$ form actually two different branches of the same unique curve, namely, $\left(\kappa-\epsilon_{\nu}\right)^{2}=\left(\epsilon_{\nu}-\lambda\right)^{2}-\tilde{m}_{\mu}^{2}$.

\section{APPENDIX B: EXTENSION OF THE FORMULAS TO THE SuSAM* MODEL}

As discussed in Secs. IV and V B, all the above analytical formulas obtained for the RFG model in the Appendix A can be used for the SuSAM* model by just replacing $\epsilon_{F} \rightarrow \epsilon_{F}^{\prime}$ and $\eta_{F} \rightarrow \eta_{F}^{\prime} \equiv \sqrt{\epsilon_{F}^{\prime 2}-1}$.

In this Appendix B we demonstrate that $\kappa_{-}^{\mathrm{NPB}}(0)=0$ and $\kappa_{+}^{\mathrm{NPB}}(0)>\eta_{F}$ for the SuSAM* model, as stated in Sec. IV [122].

It is straightforward to prove that $\kappa_{-}^{2}(0)=t_{-}(0)=0$, either from Eqs. (A7) or (A11), irrespective of the values taken by $\epsilon_{F}$ or $\epsilon_{F}^{\prime}$, provided that both are greater than 1 , as it is the case. For the second demonstration we have, from Eq. (A7),

$$
\begin{aligned}
\kappa_{+}^{2}(0)=t_{+}(0) & =\frac{\epsilon_{F}^{\prime 2}-1+\sqrt{\left(\epsilon_{F}^{\prime 2}-1\right)^{2}}}{2} \\
& =\epsilon_{F}^{\prime 2}-1 \\
& =\left(1+\left(\epsilon_{F}-1\right) \psi_{\text {left }}^{2}\right)^{2}-1,
\end{aligned}
$$

where $\psi_{\text {left }}$ is the negative value of the scaling variable, lesser than -1 , that one has to take to ensure that the scaling function is negligible beyond that value. Finally, as $\psi_{\text {left }}^{2}>1$, it is also true that

$$
\begin{aligned}
1+ & \left(\epsilon_{F}-1\right) \psi_{\text {left }}^{2}>\epsilon_{F}>1 \\
& \Rightarrow \kappa_{+}^{2}(0)=\left(1+\left(\epsilon_{F}-1\right) \psi_{\text {left }}^{2}\right)^{2}-1>\epsilon_{F}^{2}-1 \equiv \eta_{F}^{2} \\
& \Leftrightarrow \kappa_{+}(0)>\eta_{F},
\end{aligned}
$$

where in the first step we have multiplied the inequality $\psi_{\text {left }}^{2}>1$ on both sides by $\left(\epsilon_{F}-1\right)$ without changing the direction of the inequality because $\epsilon_{F}>1$.
[1] L. Alvarez-Ruso, Y. Hayato, and J. Nieves, Progress and open questions in the physics of neutrino cross sections at intermediate energies, New J. Phys. 16, 075015 (2014).

[2] K. G. Balasi, K. Langanke, and G. Martínez-Pinedo, Neutrino-nucleus reactions and their role for supernova dynamics and nucleosynthesis, Prog. Part. Nucl. Phys. 85, 33 (2015).

[3] U. Mosel, Neutrino interactions with nucleons and nuclei: Importance for long-baseline experiments, Annu. Rev. Nucl. Part. Sci. 66, 171 (2016). 
[4] T. Katori and M. Martini, Neutrino-nucleus cross sections for oscillation experiments, J. Phys. G 45, 013001 (2018).

[5] L. Alvarez-Ruso et al. (NuSTEC Collaboration), NuSTEC white paper: Status and challenges of neutrino-nucleus scattering, Prog. Part. Nucl. Phys. 100, 1 (2018).

[6] O. Benhar, P. Huber, C. Mariani, and D. Meloni, Neutrinonucleus interactions and the determination of oscillation parameters, Phys. Rep. 700, 1 (2017).

[7] C. Giusti and M. V. Ivanov, Neutral current neutrino-nucleus scattering: Theory, J. Phys. G 47, 024001 (2020).

[8] J. E. Amaro, M. B. Barbaro, J. A. Caballero, R. GonzálezJiménez, G. D. Megias, and I. Ruiz Simo, Electron- versus neutrino-nucleus scattering, J. Phys. G 47, 124001 (2020).

[9] M. Sajjad Athar and J. G. Morfin, Neutrino(Antineutrino)nucleus interactions in the shallow- and deep-inelastic scattering regions, J. Phys. G 48, 034001 (2021).

[10] P. Coloma, I. Esteban, M. C. Gonzalez-Garcia, and J. Menendez, Determining the nuclear neutron distribution from coherent elastic neutrino-nucleus scattering: Current results and future prospects, J. High Energy Phys. 08 (2020) 030.

[11] T. Kitagaki et al., High-energy quasielastic $\nu_{\mu} n \rightarrow \mu^{-} p$ scattering in deuterium, Phys. Rev. D 28, 436 (1983).

[12] S. V. Belikov et al., Quasielastic neutrino and antineutrinos scattering: Total cross-sections, axial-vector form-factor, Z. Phys. A 320, 625 (1985).

[13] K. Abe et al. (T2K Collaboration), The T2K experiment, Nucl. Instrum. Methods Phys. Res., Sect. A 659, 106 (2011).

[14] K. Abe et al. (T2K Collaboration), Precise Measurement of the Neutrino Mixing Parameter $\theta_{23}$ from Muon Neutrino Disappearance in an Off-Axis Beam, Phys. Rev. Lett. 112, 181801 (2014).

[15] K. Abe et al. (T2K Collaboration), Measurement of the $\nu_{\mu}$ charged-current quasielastic cross section on carbon with the ND280 detector at T2K, Phys. Rev. D 92, 112003 (2015).

[16] K. Abe et al. (T2K Collaboration), Measurement of the $\nu_{\mu}$ charged current quasielastic cross section on carbon with the T2K on-axis neutrino beam, Phys. Rev. D 91, 112002 (2015).

[17] K. Abe et al. (T2K Collaboration), Measurement of the muon neutrino inclusive charged-current cross section in the energy range of 1-3 GeV with the T2K INGRID detector, Phys. Rev. D 93, 072002 (2016).

[18] Y. Nakajima et al. (SciBooNE Collaboration), Measurement of inclusive charged current interactions on Carbon in a few-GeV neutrino beam, Phys. Rev. D 83, 012005 (2011).

[19] J. L. Alcaraz-Aunion and J. Walding (SciBooNE Collaboration), Measurement of the $\nu_{\mu}$-CCQE cross section in the SciBooNE experiment, AIP Conf. Proc. 1189, 145 (2009).

[20] A. A. Aguilar-Arevalo et al. (MiniBooNE Collaboration), First Measurement of Monoenergetic Muon Neutrino Charged Current Interactions, Phys. Rev. Lett. 120, 141802 (2018).

[21] A. A. Aguilar-Arevalo et al. (MiniBooNE Collaboration), First measurement of the muon antineutrino doubledifferential charged-current quasielastic cross section, Phys. Rev. D 88, 032001 (2013).
[22] A. A. Aguilar-Arevalo et al. (MiniBooNE Collaboration), First measurement of the muon neutrino charged current quasielastic double differential cross section, Phys. Rev. D 81, 092005 (2010).

[23] A. A. Aguilar-Arevalo et al. (MiniBooNE Collaboration), Measurement of Muon Neutrino Quasielastic Scattering on Carbon, Phys. Rev. Lett. 100, 032301 (2008).

[24] P. Adamson et al. (MINOS Collaboration), Study of quasielastic scattering using charged-current $\nu_{\mu}$-iron interactions in the MINOS near detector, Phys. Rev. D 91, 012005 (2015).

[25] M. F. Carneiro et al. (MINER $\nu$ A Collaboration), HighStatistics Measurement of Neutrino Quasielasticlike Scattering at $6 \mathrm{GeV}$ on a Hydrocarbon Target, Phys. Rev. Lett. 124, 121801 (2020).

[26] D. Ruterbories et al. (MINER $\nu$ A Collaboration), Measurement of quasielastic-like neutrino scattering at $\left\langle E_{\nu}\right\rangle \sim$ 3.5 GeV on a hydrocarbon target, Phys. Rev. D 99, 012004 (2019).

[27] C. E. Patrick et al. (MINER $\nu$ A Collaboration), Measurement of the muon antineutrino double-differential cross section for quasielastic-like scattering on hydrocarbon at $E_{\nu} \sim 3.5 \mathrm{GeV}$, Phys. Rev. D 97, 052002 (2018).

[28] J. Wolcott et al. (MINER $\nu$ A Collaboration), Measurement of Electron Neutrino Quasielastic and Quasielasticlike Scattering on Hydrocarbon at $\left\langle E_{\nu}\right\rangle=3.6 \mathrm{GeV}$, Phys. Rev. Lett. 116, 081802 (2016).

[29] G. A. Fiorentini et al. (MINER $\nu$ A Collaboration), Measurement of Muon Neutrino Quasielastic Scattering on a Hydrocarbon Target at $E_{\nu} \sim 3.5 \mathrm{GeV}$, Phys. Rev. Lett. 111, 022502 (2013).

[30] L. Fields et al. (MINER $\nu$ A Collaboration), Measurement of Muon Antineutrino Quasielastic Scattering on a Hydrocarbon Target at $E_{\nu} \sim 3.5 \mathrm{GeV}$, Phys. Rev. Lett. 111, 022501 (2013).

[31] P. Abratenko et al. (MicroBooNE Collaboration), First Measurement of Differential Charged Current Quasielasticlike $\nu_{\mu}$-Argon Scattering Cross Sections with the MicroBooNE Detector, Phys. Rev. Lett. 125, 201803 (2020).

[32] R. Acciarri et al. (ArgoNeuT Collaboration), First measurement of electron neutrino scattering cross section on argon, Phys. Rev. D 102, 011101 (2020).

[33] R. Acciarri et al. (ArgoNeuT Collaboration), Measurements of inclusive muon neutrino and antineutrino charged current differential cross sections on Argon in the NuMI antineutrino beam, Phys. Rev. D 89, 112003 (2014).

[34] P. Adamson et al. ( $\mathrm{NO} \nu \mathrm{A}$ Collaboration), Constraints on Oscillation Parameters from $\nu_{e}$ Appearance and $\nu_{\mu}$ Disappearance in NO $\nu$ A, Phys. Rev. Lett. 118, 231801 (2017).

[35] A. M. Ankowski, High-energy limit of neutrino quasielastic cross section, Acta Phys. Pol. B 37, 377 (2006).

[36] A. M. Ankowski, How different can the $\nu_{\mu}$ and $\nu_{e}$ cross sections be?, in Proceedings of the 20th International Workshop on Neutrinos from Accelerators (NUFACT 2018), Blacksburg, Virginia, USA, 2018 (Proceedings of Science, SISSA, Trieste,2019), Vol. NuFACT2018, p. 092.

[37] K. Abe et al. (T2K Collaboration), Constraint on the matter-antimatter symmetry-violating phase in neutrino 
oscillations, Nature (London) 580, 339 (2020); 583, E16 (E) (2020).

[38] R. Acciarri et al. (DUNE Collaboration), Long-Baseline Neutrino Facility (LBNF) and Deep Underground Neutrino Experiment (DUNE). Conceptual design report. Volume 2: The physics program for DUNE at LBNF, arXiv: 1512.06148 .

[39] A. M. Ankowski and C. Mariani, Systematic uncertainties in long-baseline neutrino-oscillation experiments, J. Phys. G 44, 054001 (2017).

[40] A. M. Ankowski, Effect of the charged-lepton's mass on the quasielastic neutrino cross sections, Phys. Rev. C 96, 035501 (2017).

[41] M. Martini, N. Jachowicz, M. Ericson, V. Pandey, T. Van Cuyck, and N. Van Dessel, Electron-neutrino scattering off nuclei from two different theoretical perspectives, Phys. Rev. C 94, 015501 (2016).

[42] A. Nikolakopoulos, N. Jachowicz, N. Van Dessel, K. Niewczas, R. González-Jiménez, J. M. Udías, and V. Pandey, Electron Versus Muon Neutrino Induced Cross Sections in Charged Current Quasielastic Processes, Phys. Rev. Lett. 123, 052501 (2019).

[43] W. M. Alberico and A. Molinari, Relativistic response of a Fermi gas, J. Phys. G 7, L93 (1981).

[44] R. A. Smith and E. J. Moniz, Neutrino reactions on nuclear targets, Nucl. Phys. B43, 605 (1972); 101, 547(E) (1975).

[45] E. J. Moniz, I. Sick, R. R. Whitney, J. R. Ficenec, R. D. Kephart, and W. P. Trower, Nuclear Fermi Momenta from Quasielastic Electron Scattering, Phys. Rev. Lett. 26, 445 (1971).

[46] E. J. Moniz, Pion electroproduction from nuclei, Phys. Rev. 184, 1154 (1969).

[47] K. S. Kuzmin, V. V. Lyubushkin, and V. A. Naumov, Quasielastic axial-vector mass from experiments on neutrino-nucleus scattering, Eur. Phys. J. C 54, 517 (2008).

[48] J. E. Amaro, E. Ruiz Arriola, and I. Ruiz Simo, Scaling violation and relativistic effective mass from quasi-elastic electron scattering: Implications for neutrino reactions, Phys. Rev. C 92, 054607 (2015); 100, 019904(E) (2019).

[49] J. E. Amaro, E. Ruiz Arriola, and I. Ruiz Simo, Superscaling analysis of quasielastic electron scattering with relativistic effective mass, Phys. Rev. D 95, 076009 (2017).

[50] V. L. Martinez-Consentino, I. Ruiz Simo, J. E. Amaro, and E. Ruiz Arriola, Fermi-momentum dependence of relativistic effective mass below saturation from superscaling of quasielastic electron scattering, Phys. Rev. C 96, 064612 (2017).

[51] I. Ruiz Simo, V. L. Martinez-Consentino, J. E. Amaro, and E. Ruiz Arriola, Quasielastic charged-current neutrino scattering in the scaling model with relativistic effective mass, Phys. Rev. D 97, 116006 (2018).

[52] J. E. Amaro, V. L. Martinez-Consentino, E. Ruiz Arriola, and I. Ruiz Simo, Global superscaling analysis of quasielastic electron scattering with relativistic effective mass, Phys. Rev. C 98, 024627 (2018).

[53] W. M. Alberico, A. Molinari, T. W. Donnelly, E. L. Kronenberg, and J.W. Van Orden, Scaling in electron scattering from a relativistic Fermi gas, Phys. Rev. C 38, 1801 (1988).
[54] M. B. Barbaro, J. A. Caballero, T. W. Donnelly, and C. Maieron, Inelastic electron nucleus scattering and scaling at high inelasticity, Phys. Rev. C 69, 035502 (2004).

[55] M. B. Barbaro, R. Cenni, A. De Pace, T. W. Donnelly, and A. Molinari, Relativistic $y$-scaling and the Coulomb sum rule in nuclei, Nucl. Phys. A643, 137 (1998).

[56] D. B. Day, J. S. McCarthy, T. W. Donnelly, and I. Sick, Scaling in inclusive electron-nucleus scattering, Annu. Rev. Nucl. Part. Sci. 40, 357 (1990).

[57] J. A. Caballero, J. E. Amaro, M. B. Barbaro, T. W. Donnelly, and J.M. Udías, Scaling and isospin effects in quasielastic lepton-nucleus scattering in the Relativistic Mean Field Approach, Phys. Lett. B 653, 366 (2007).

[58] F. Sanchez (private communication).

[59] J. E. Amaro, M. B. Barbaro, J. A. Caballero, T. W. Donnelly, A. Molinari, and I. Sick, Using electron scattering superscaling to predict charge-changing neutrino cross sections in nuclei, Phys. Rev. C 71, 015501 (2005).

[60] J. E. Amaro, M. B. Barbaro, J. A. Caballero, T. W. Donnelly, and C. Maieron, Semi-relativistic description of quasielastic neutrino reactions and superscaling in a continuum shell model, Phys. Rev. C 71, 065501 (2005).

[61] J. E. Amaro and E. Ruiz Arriola, Axial-vector dominance predictions in quasielastic neutrino-nucleus scattering, Phys. Rev. D 93, 053002 (2016).

[62] Notice that in Eq. (1) we have particularized the general expression for the scattering of muon neutrinos/antineutrinos, but the expression is general for other kind of neutrino species, just by changing the final lepton mass.

[63] C. Maieron, T. W. Donnelly, and I. Sick, Extended superscaling of electron scattering from nuclei, Phys. Rev. C 65 , 025502 (2002).

[64] R. González-Jiménez, G. D. Megias, M. B. Barbaro, J. A. Caballero, and T. W. Donnelly, Extensions of superscaling from relativistic mean field theory: The SuSAv2 model, Phys. Rev. C 90, 035501 (2014).

[65] If the kind of neutrino is a distinct one, one can use the formulas developed in Secs. III B and Appendix A 4 and change them for other final charged lepton masses.

[66] J. D. Walecka, A theory of highly condensed matter, Ann. Phys. (N.Y.) 83, 491 (1974).

[67] B. D. Serot and J.D. Walecka, The relativistic nuclear many body problem, Adv. Nucl. Phys. 16, 1 (1986).

[68] K. Wehrberger, Electromagnetic response functions in quantum hadrodynamics, Phys. Rep. 225, 273 (1993).

[69] R. Rosenfelder, Quasielastic electron scattering from nuclei, Ann. Phys. (N.Y.) 128, 188 (1980).

[70] O. Benhar, D. B. Day, and I. Sick, An archive for quasielastic electron-nucleus scattering data, arXiv:nucl-ex/ 0603032.

[71] O. Benhar, D. B. Day, and I. Sick, Inclusive quasielastic electron-nucleus scattering, Rev. Mod. Phys. 80, 189 (2008).

[72] I. Ruiz Simo, R. Navarro Pérez, J. E. Amaro, and E. Ruiz Arriola, Coarse graining the Bethe-Goldstone equation: Nucleon-nucleon high-momentum components, Phys. Rev. C 96, 054006 (2017).

[73] I. Sick, D. B. Day, and J. S. McCarthy, Nuclear High Momentum Components and $y$ Scaling in Electron Scattering, Phys. Rev. Lett. 45, 871 (1980). 
[74] A. Ramos, A. Polls, and W. H. Dickhoff, Single-particle properties and short-range correlations in nuclear matter, Nucl. Phys. A503, 1 (1989).

[75] J. Arrington, D. W. Higinbotham, G. Rosner, and M. Sargsian, Hard probes of short-range nucleon-nucleon correlations, Prog. Part. Nucl. Phys. 67, 898 (2012).

[76] H. Müther, A. Polls, and W. H. Dickhoff, Momentum and energy distributions of nucleons in finite nuclei due to short-range correlations, Phys. Rev. C 51, 3040 (1995).

[77] C. Giusti, H. Müther, F. D. Pacati, and M. Stauf, Shortrange and tensor correlations in the ${ }^{16} \mathrm{O}\left(e, e^{\prime} p n\right)$ reaction, Phys. Rev. C 60, 054608 (1999).

[78] M. V. Stoitsov, A. N. Antonov, and S. S. Dimitrova, Natural orbital representation and short-range correlations in nuclei, Phys. Rev. C 48, 74 (1993).

[79] M. Alvioli, C. Ciofi degli Atti, L. P. Kaptari, C. B. Mezzetti, and H. Morita, Nucleon momentum distributions, their spin-isospin dependence, and short-range correlations, Phys. Rev. C 87, 034603 (2013).

[80] M. Vanhalst, J. Ryckebusch, and W. Cosyn, Quantifying short-range correlations in nuclei, Phys. Rev. C 86, 044619 (2012).

[81] T. Van Cuyck, N. Jachowicz, R. González-Jiménez, M. Martini, V. Pandey, J. Ryckebusch, and N. Van Dessel, Influence of short-range correlations in neutrino-nucleus scattering, Phys. Rev. C 94, 024611 (2016).

[82] N. Fomin, D. Higinbotham, M. Sargsian, and P. Solvignon, New results on short-range correlations in nuclei, Annu. Rev. Nucl. Part. Sci. 67, 129 (2017).

[83] J.E. Amaro, A. M. Lallena, G. Co', and A. Fabrocini, Model of short-range correlations in the charge response, Phys. Rev. C 57, 3473 (1998).

[84] M. Mazziotta, J.E. Amaro, and F. Arias de Saavedra, Effects of short-range correlations in $\left(e, e^{\prime} p\right)$ reactions and nuclear overlap functions, Phys. Rev. C 65, 034602 (2002).

[85] W. Weise, Effects of short-range correlations on the quasielastic scattering of electrons, Nucl. Phys. A193, 625 (1972).

[86] I. Ruiz Simo, R. Navarro Pérez, J.E. Amaro, and E. Ruiz Arriola, Coarse-grained short-range correlations, Phys. Rev. C 95, 054003 (2017).

[87] R. B. Wiringa, R. Schiavilla, S. C. Pieper, and J. Carlson, Nucleon and nucleon-pair momentum distributions in $A \leq 12$ nuclei, Phys. Rev. C 89, 024305 (2014).

[88] R. Schiavilla, D. S. Lewart, V. R. Pandharipande, S. C. Pieper, R. B. Wiringa, and S. Fantoni, Structure functions and correlations in nuclei, Nucl. Phys. A473, 267 (1987).

[89] D. Berardo, M. B. Barbaro, R. Cenni, T. W. Donnelly, and A. Molinari, Connecting scaling with short-range correlations, Phys. Rev. C 84, 054315 (2011).

[90] V. Tornow, D. Drechsel, G. Orlandini, and M. Traini, Effects of wave function correlations on scaling violation in quasi-free electron scattering, Phys. Lett. B 107, 259 (1981).

[91] D. Day, Short range correlations, inclusive electronnucleus scattering, and scaling, AIP Conf. Proc. 1056, 315 (2008).

[92] V. L. Martinez-Consentino, I. R. Simo, and J.E. Amaro, Meson-exchange currents and superscaling analysis with relativistic effective mass of quasielastic electron scattering from ${ }^{12}$ C, Phys. Rev. C 104, 025501 (2021).

[93] G. D. Megias, M. V. Ivanov, R. González-Jiménez, M. B. Barbaro, J. A. Caballero, T. W. Donnelly, and J. M. Udías, Nuclear effects in neutrino and antineutrino chargedcurrent quasielastic scattering at MINER $\nu$ A kinematics, Phys. Rev. D 89, 093002 (2014); 91, 039903(E) (2015).

[94] The case of $\tau$ neutrinos should be taken with care and studied in depth because both solutions could be positive if the nucleon mass were the relativistic effective mass of the Walecka model [66-69], which is the underlying theoretical model in which the SuSAM* is based on.

[95] S. Dolan, G. D. Megias, and S. Bolognesi, Implementation of the SuSAv2-meson exchange current $1 \mathrm{p} 1 \mathrm{~h}$ and $2 \mathrm{p} 2 \mathrm{~h}$ models in GENIE and analysis of nuclear effects in T2K measurements, Phys. Rev. D 101, 033003 (2020).

[96] G. D. Megias, J. E. Amaro, M. B. Barbaro, J. A. Caballero, T. W. Donnelly, and I. Ruiz Simo, Charged-current neutrinonucleus reactions within the superscaling meson-exchange current approach, Phys. Rev. D 94, 093004 (2016).

[97] V. V. Lyubushkin et al. (NOMAD Collaboration), A study of quasi-elastic muon neutrino and antineutrino scattering in the NOMAD experiment, Eur. Phys. J. C 63, 355 (2009).

[98] G. D. Megias, J. E. Amaro, M. B. Barbaro, J. A. Caballero, and T. W. Donnelly, Inclusive electron scattering within the SuSAv2 meson-exchange current approach, Phys. Rev. D 94, 013012 (2016).

[99] I. Ruiz Simo, J. E. Amaro, M. B. Barbaro, A. De Pace, J. A. Caballero, and T. W. Donnelly, Relativistic model of $2 \mathrm{p}-2 \mathrm{~h}$ meson exchange currents in (anti)neutrino scattering, J. Phys. G 44, 065105 (2017).

[100] A. V. Butkevich, Analysis of quasi-elastic neutrino charged-current scattering off ${ }^{16} \mathrm{O}$ and neutrino energy reconstruction, Phys. Rev. C 78, 015501 (2008).

[101] M. Martini, M. Ericson, and G. Chanfray, Neutrino energy reconstruction problems and neutrino oscillations, Phys. Rev. D 85, 093012 (2012).

[102] J. Nieves, F. Sánchez, I. Ruiz Simo, and M. J. Vicente Vacas, Neutrino energy reconstruction and the shape of the charged current quasielastic-like total cross section, Phys. Rev. D 85, 113008 (2012).

[103] M. Martini, M. Ericson, and G. Chanfray, Energy reconstruction effects in neutrino oscillation experiments and implications for the analysis, Phys. Rev. D 87, 013009 (2013).

[104] T. Leitner and U. Mosel, Neutrino-nucleus scattering reexamined: Quasielastic scattering and pion production entanglement and implications for neutrino energy reconstruction, Phys. Rev. C 81, 064614 (2010).

[105] A. M. Ankowski, O. Benhar, and M. Sakuda, Improving the accuracy of neutrino energy reconstruction in chargedcurrent quasielastic scattering off nuclear targets, Phys. Rev. D 91, 033005 (2015).

[106] U. Mosel, O. Lalakulich, and K. Gallmeister, Energy Reconstruction in the Long-Baseline Neutrino Experiment, Phys. Rev. Lett. 112, 151802 (2014).

[107] V. De Romeri, E. Fernandez-Martinez, and M. Sorel, Neutrino oscillations at DUNE with improved energy reconstruction, J. High Energy Phys. 09 (2016) 030. 
[108] X.-G. Lu, D. Coplowe, R. Shah, G. Barr, D. Wark, and A. Weber, Reconstruction of energy spectra of neutrino beams independent of nuclear effects, Phys. Rev. D 92, 051302 (2015).

[109] A. M. Ankowski, O. Benhar, P. Coloma, P. Huber, C.-M. Jen, C. Mariani, D. Meloni, and E. Vagnoni, Comparison of the calorimetric and kinematic methods of neutrino energy reconstruction in disappearance experiments, Phys. Rev. D 92, 073014 (2015).

[110] L. Munteanu, S. Suvorov, S. Dolan, D. Sgalaberna, S. Bolognesi, S. Manly, G. Yang, C. Giganti, K. Iwamoto, and C. Jesús-Valls, New method for an improved antineutrino energy reconstruction with charged-current interactions in next-generation detectors, Phys. Rev. D 101, 092003 (2020).

[111] A. P. Furmanski and J. T. Sobczyk, Neutrino energy reconstruction from one-muon and one-proton events, Phys. Rev. C 95, 065501 (2017).

[112] S. Galster, H. Klein, J. Moritz, K. H. Schmidt, D. Wegener, and J. Bleckwenn, Elastic electron-deuteron scattering and the electric neutron form factor at four-momentum transfers $5 \mathrm{fm}^{-2}<q^{2}<14 \mathrm{fm}^{-2}$, Nucl. Phys. B32, 221 (1971).

[113] I. D. Kakorin, K. S. Kuzmin, and V. A. Naumov, A unified empirical model for quasielastic interactions of neutrino and antineutrino with nuclei, Phys. Part. Nucl. Lett. 17, 265 (2020).

[114] N. Suwonjandee, The measurement of the quasi-elastic neutrino-nucleon scattering cross section at the Tevatron, Ph.D. thesis, Cincinnati University, 2004.

[115] Note that $\epsilon_{0}=\kappa \sqrt{1+1 / \tau}-\lambda$ can be also hold in the region where $\kappa<\eta_{F}$ if $\kappa \sqrt{1+1 / \tau}>\epsilon_{F}-\lambda$ (see Eq. (A12) and below).
[116] This statement can be rigorously and mathematically proved, but the easiest way to convince any reader of it is to have a look at Fig. 1 of Ref. [53].

[117] I. Ruiz Simo, J. E. Amaro, M. B. Barbaro, J. A. Caballero, G. D. Megias, and T.W. Donnelly, Two-nucleon emission in neutrino and electron scattering from nuclei: The modified convolution approximation, Ann. Phys. (Amsterdam) 388, 323 (2018).

[118] The other possibility, i.e., that $\lambda \geq \lambda_{+}>\lambda_{-}$can be ruled out because then $\lambda \geq\left(\epsilon_{F}+1\right) / 2>1>\eta_{F} \equiv k_{F} / m_{N}$ (even with effective nucleon masses as in the SuSAM* model, we will always have that the Fermi momentum is smaller than the nucleon mass, regardless of this mass being the free nucleon mass or the relativistic effective one), and we are seeking solutions in the region where $\lambda<\eta_{F}$.

[119] This last feature can be stated because the discriminant of Eq. (A15) is positive and lesser than $\rho^{2}$. Thus the square root of the discriminant is also lesser than $\rho$, and then $u_{-}(\lambda)$ is necessarily positive in the region where $0 \leq \lambda \leq \lambda_{-}$.

[120] Another way to arrive to the same solution would have been to solve $\kappa_{+}^{\mathrm{NPB}}(0)=\kappa_{\max }^{\text {lepton }}(0)$ for $\epsilon_{\nu}$. As $\kappa_{+}^{\mathrm{NPB}}(0)=\eta_{F}$ (see Eq. (A5), and $\kappa_{\max }^{\text {lepton }}(0)=\epsilon_{\nu}+\sqrt{\epsilon_{\nu}^{2}-\tilde{m}_{\mu}^{2}}$ (see definition given in (14)), the solution $\epsilon_{\nu_{+}}$would have been obtained in a much simpler way.

[121] The reason for this statement is because $\kappa_{\max }^{\text {lepton }}(\lambda)$ is monotonically decreasing with $\lambda$ and $\kappa_{-}^{\mathrm{NPB}}(\lambda)$ is a monotonically increasing function of $\lambda$, but smaller than $\kappa_{+}^{\mathrm{NPB}}(\lambda)$.

[122] For the demonstrations provided here, we drop the label NPB from all the expressions in order to shorten the already cumbersome notation. 